\title{
Heat and fluid flow in a scraped-surface heat exchanger containing a fluid with temperature-dependent viscosity
}

\author{
A. A. T. Smith, S. K. Wilson*, B. R. Duffy ${ }^{\dagger}$ \\ Department of Mathematics and Statistics, \\ University of Strathclyde, \\ Livingstone Tower, \\ 26 Richmond Street, \\ Glasgow G1 1XH, \\ United Kingdom \\ and N. Hall-Taylor \\ Chemtech International Limited, \\ 448 Basingstoke Road, \\ Reading RG2 0LP, \\ United Kingdom
}

22nd December 2009, revised 23rd and 27th July 2010

\footnotetext{
${ }^{*}$ Corresponding Author. Telephone: + 44 (0) 141548 3820, Fax: + 44 (0) 141548 3345, Email: s.k.wilson@strath.ac.uk

${ }^{\dagger}$ Email: b.r.duffy@strath.ac.uk

Email: Nick@chemtechinternational.com
} 


\begin{abstract}
Scraped-surface heat exchangers (SSHEs) are extensively used in a wide variety of industrial settings where the continuous processing of fluids and fluid-like materials is involved. The steady non-isothermal flow of a Newtonian fluid with temperature-dependent viscosity in a narrow-gap SSHE when a constant temperature difference is imposed across the gap between the rotor and the stator is investigated. The mathematical model is formulated and the exact analytical solutions for the heat and fluid flow of a fluid with a general dependence of viscosity on temperature for a general blade shape are obtained. These solutions are then presented for the specific case of an exponential dependence of viscosity on temperature. Asymptotic methods are employed to investigate the behaviour of the solutions in several special limiting geometries and in the limits of weak and strong thermoviscosity. In particular, in the limit of strong thermoviscosity (i.e. strong heating or cooling and/or strong dependence of viscosity on temperature) the transverse and axial velocities become uniform in the bulk of the flow with boundary layers forming either just below the blade and just below the stationary upper wall or just above the blade and just above the moving lower wall. Results are presented for the most realistic case of a linear blade which illustrate the effect of varying the thermoviscosity of the fluid and the geometry of the SSHE on the flow.
\end{abstract}

Keywords: Scraped-surface heat exchanger, temperature-dependent viscosity, thermoviscosity, lubrication approximation, asymptotic methods 


\section{Introduction}

Scraped-surface heat exchangers (SSHEs) are extensively used in a wide variety of industrial settings where the continuous processing of fluids and fluid-like materials is involved. They are often used in the pharmaceutical and chemical industries (for example, in dewaxing oils and producing paints); however, they are most commonly found within the food manufacturing sector, where they are used for mixing and heating or cooling foodstuffs during processes such as sterilisation, crystallisation and gelatinisation. Unlike the simpler plate heat exchangers which are commonly used for low viscosity process fluids, SSHEs are designed to deal with the problems that arise when processing very viscous products. Foodstuffs such as margarine, ice-cream, chocolate, sauces, spreads, peanut butter, cream, caramel, purées, soups, salad dressings, yoghurt and jam are all routinely processed using SSHEs. The short article by Smith [1] gives a good introduction to the applicability of and differences between plate heat exchangers and SSHEs.

An SSHE basically consists of a cylindrical rotating shaft (the "rotor") within a concentric hollow stationary cylinder (the "stator") so as to form an annular region along which the process fluid is pumped. The stator acts as the heat-transfer surface, and it is normally enclosed within another cylindrical tube which provides a gap through which a heating or cooling service fluid (for example, steam or ammonia) passes. Attached to the rotor are a number of pivoted blades each of which scrapes the heat-transfer surface, removing processed fluid and hence allowing unprocessed fluid to come closer to the stator. A cut-away schematic of a typical SSHE is shown in Fig. 1. For maximum efficiency the stator is manufactured from a material with a high heat-transfer coefficient (such as nickel) though it is usually coated with a hard chrome-plated finish in order to protect it from the scraping action of the blades. The blades themselves are usually made from stainless steel; however, plastic ones are used for certain special applications (and in these applications the chrome plating is not necessarily required) [1]. In a typical SSHE there are usually either two or four blades located periodically around the rotor, and usually these extend the whole way down the length of the device, though sometimes they are axially staggered in order to improve mixing. More advanced designs of SSHE include features such as holes in the blades (which reduce power consumption), oval stators (which reduce "channelling" in which fluid passes through the exchanger relatively unprocessed), and non-centrally mounted shafts (which enhance mixing and prevent material from building up on the underside of the blades).

The rotating scraper blades serve several purposes which are particularly beneficial in food processing. As we have already described, their main benefit is that they improve the heat transfer between the stator and the process fluid by continually replacing the fluid closest to the stator. This ensures that the fluid is more evenly processed, thereby reducing the chances of it having temperature inhomogeneities when it exits the SSHE (essential for processes such as sterilisation). Since the stator is being scraped, the problem of decreased efficiency in heat transfer due to food deposits accumulating on the heat-transfer surface is also avoided, which means that the SSHE can be run continuously for longer periods of time. The blades also serve to mix the material, which often helps to produce a more desirable consistent quality to the texture and taste of the product. In the manufacture of ice-cream, for example, the action of the blades helps to blend the fluid, air and ice crystals that are formed on the cooled stator surface, to produce a smooth consistency. This combination of mixing the fluid while heating or cooling 
also means that high temperature gradients can be utilised without compromising the product.

On the downside, the more complex structure of an SSHE makes it a more expensive capital investment than some conventional heat exchangers and so, as a result, food manufacturers are naturally interested in being able to optimise their production runs to reduce operating costs. For these reasons, much experimental and theoretical research has been carried out on a variety of types of SSHE using different operating conditions and fluid rheologies. Due to the complicated nature of the heating/cooling and mixing process, there are several factors that need to be taken into account in order to develop a fuller understanding of the flow within an SSHE. First, the geometry and operating parameters of the SSHE will obviously play a major role, and properties such as device dimensions, rotation speeds, blade design and flow rates need to be carefully considered in order to maximise efficiency and create the desired conditions within the heat exchanger. Secondly, the flow is three-dimensional (comprising a transverse flow driven by the moving rotor and blades and a pressure-driven axial flow) and in certain circumstances it can be complicated by the formation of vortices (see Trommelen and Beek [2], Härröd [3] and Dumont, Fayolle and Legrand [4]). The flow is also non-isothermal and will typically involve conduction, convection and viscous dissipation within the process fluid; moreover, the thermal characteristics of the service fluid may be significant. The other major factors that must be taken into account are the rheological characteristics of the process fluid. As previously mentioned, SSHEs are typically used for highly viscous fluids; however, the fluids that are processed are often non-Newtonian, inhomogeneous, viscoplastic, viscoelastic, contain particulates, possess temperature-dependent viscosities, and/or undergo phase changes during the cycle through the device. Clearly this wide range of fluid properties will lead to a variety of flow behaviour. Hall-Taylor [5] gives a general overview of SSHE applications in food processing and the various factors that need to be taken into account.

A search of the literature reveals a fairly extensive body of experimental research on SSHEs. Flow of a non-Newtonian fluid under isothermal conditions has been investigated by, for example, Russell, Burmester and Winch [6], Wang, Walton and McCarthy [7] and Stranzinger, Feigl and Windhab [8]. Heat-transfer mechanisms have been studied by Trommelen, Beek and Van de Westelaken [9] and Qin, Chen and Russell [10], amongst others, and power consumption has been analysed by Trommelen and Beek [11]. In order to analyse fully a mathematical model for heat and fluid flow in an SSHE it is ultimately necessary to resort to numerical methods. Sun et al. [12] and Baccar and Abid $[13,14]$ performed numerical simulations involving fluid flow and heat transfer in the context of SSHEs, while related work by Sun et al. [15] investigated isothermal flow of shear-thinning fluids in lid-driven cavities in the presence of an axial throughflow. Although these numerical approaches are useful in that they provide a more detailed description of the behaviour, they can fail to elucidate the subtle interplay that the various device parameters and fluid properties can have on the solutions. In order to develop a better understanding of some of the processes occurring within an SSHE some recent work has concentrated on specific aspects of the problem that can be studied analytically. Fitt and Please [16] modelled isothermal flow of a shear-thinning fluid in a simplified model of a narrow-gap SSHE which allowed them to determine the optimal power distribution between rotation and pumping. Duffy, Wilson and Lee [17] developed a mathematical model for isothermal flow of a Newtonian fluid in a narrow-gap SSHE 
and obtained analytical expressions for the velocities, pressures and volume fluxes, and for the forces on the device. The latter authors also calculated the possible equilibrium positions of the blades and found that the blades can make the desired contact with the stator when their pivots are located sufficiently close to the end of the blades. In an accompanying paper Fitt, Lee and Please [18] investigated the phenomenon of channelling of a Newtonian fluid in a simplified model of a narrow-gap SSHE. Very recently Rodriguez Pascual et al. [19] visualised the flow within a laboratory-scale SSHE and found qualitative agreement with numerical simulations obtained using a lattice-Boltzmann discretization to solve the Navier-Stokes equations, and a Lagrangian approach to particle tracking. For further information on experimental and theoretical work on SSHEs the reader is referred to the extensive literature reviews by Härröd [20] (published over 20 years ago in 1986) and Rao and Hartel [21] (published much more recently in 2006).

The present work extends the isothermal analysis of Duffy et al. [17] to investigate the steady non-isothermal flow of a Newtonian fluid with temperature-dependent viscosity in a narrow-gap SSHE when a constant temperature difference is imposed across the gap between the rotor and the stator. In particular, we formulate a mathematical model and obtain the exact analytical solutions for the heat and fluid flow of a fluid with a general dependence of viscosity on temperature for a general blade shape. These solutions are then analysed for the specific case of an exponential dependence of viscosity on temperature and illustrated for the most realistic case of a linear blade.

\section{The Mathematical Model}

\subsection{Formulation of the Model}

Consider the steady flow of a Newtonian fluid with temperature-dependent viscosity in an SSHE such as that shown in Fig. 1 when a constant temperature difference is imposed across the gap between the rotor and the stator. The three-dimensional flow within such a device is composed of a transverse flow driven by the relative motion of the rotor and the stator and an axial flow driven by an imposed axial pressure gradient. In the present work we consider a narrow-gap SSHE, a common type of SSHE in which the annular gap between the rotor and the stator is thin relative to the circumferential length of the device. According to information supplied by Chemtech International Ltd (Reading, UK), a representative SSHE of this type has an axial length, $D$, of 2 $\mathrm{m}$, a rotor circumference of approximately $427 \mathrm{~mm}$, an annular gap, $H$, of $9 \mathrm{~mm}$ and uses two blades each of whose length, $L$, is roughly $35 \mathrm{~mm}$. These values correspond to a small transverse aspect ratio of $\epsilon=H / L \simeq 0.3$, suggesting that the problem of determining the transverse heat and fluid flow within such a device can be made analytically tractable by employing a lubrication approximation. At leading order in the limit of small transverse aspect ratio the problem for the transverse flow is that of flow in a parallel-sided channel containing a periodic array of angled blades each of which makes contact with the lower wall (i.e. the stator). The blades are not, however, connected to this wall, but are attached by rods and pins to the upper wall (i.e. the rotor). In reality it is the rotor and the attached blades that move while the stator remains fixed; however, since fluid inertia is typically negligible in practice, a Galilean shift may be implemented so that we can consider the simpler (but entirely equivalent) situation in which the lower wall moves and the upper wall and the attached blades are 
fixed. For simplicity in the present work we will neglect the effects of gravity; however, it is straightforward to include it in the axial pressure gradient if the SSHE is mounted with the axis vertical, as is often the case in practice. The viscosity of the fluid $\mu$ is taken to be a known function of temperature $T$, i.e. $\mu=\mu(T)$. A temperature difference is imposed across the gap between the rotor and stator, and the transport of heat in the fluid is assumed to be conduction dominated (i.e. we neglect convection and viscous dissipation). We assume that the blades are perfect thermal conductors and hence that both temperature and heat flux are continuous across them. As previously mentioned, the blades are often manufactured from stainless steel, which has a reasonably high thermal conductivity and, since the blades are relatively thin, it is reasonable to assume that they are perfect thermal conductors. However, for the special applications in which plastic blades are used it would be more appropriate to model them as perfect thermal insulators, and this case may be analysed in a future publication. The fluid velocity satisfies no-slip and no-penetration conditions on all solid boundaries, and the pressure is continuous throughout the channel.

Figure 2 shows the geometry of the leading order mathematical model and defines the Cartesian coordinates $O x y z$ used to describe position within the channel. A representative blade is situated between $x=0$ and $x=L$ and its shape is represented by the equation $y=h(x)$ satisfying $h(0)=0$ (so that it scrapes the moving lower wall at $x=0$ ), $0<h(x)<H$ for $0<x<L$ and $0<h(L) \leqslant H$ (so that it lies strictly below the stationary upper wall $y=H$ except possibly at $x=L$ ), where $H$ denotes the width of the channel. It is convenient to divide the flow domain into three regions: region 1 lies between the blade and the lower wall and hence occupies $0 \leqslant x<L$ and $0<y<h$, region 2 lies between the blade and the upper wall and hence occupies $0 \leqslant x<L$ and $h<y<H$, while region 3 is the space between the blades and hence occupies $L \leqslant x<L+l$ and $0<y<H$. This structure is repeated periodically in $x$ with a period of $L+l$, and so henceforth we need only consider the section of the channel between $x=0$ and $x=L+l$ containing one of each of the three regions. As described previously, we consider the situation in which the upper wall and the attached blades remain stationary while the lower wall moves in the positive $x$ direction with velocity $U$. The mathematical model will be solved for a general blade shape $h$; however, in Sec. 5 we will consider the most realistic case of a linear blade given by $h=\alpha x$, where the constant $\alpha$ (satisfying $0 \leqslant \alpha \leqslant \epsilon=H / L$ ) is the slope of the blade. The fluid is subject to a constant axial pressure gradient $-G$ and a constant temperature difference across the channel, the latter caused by prescribing the temperature $T$ to be $T=T_{0}$ on $y=0$ and $T=T_{H}$ on $y=H$.

In respect to a real SSHE, the width $H$, period $L+l$ and speed $U$ are given by $H=R_{2}-R_{1}, L+l=2 \pi R_{1} / N$ and $U=\omega R_{1}$, where $R_{1}$ and $R_{2}$ are the radii of the rotor and stator, respectively, $N$ is the number of blades in a cross-section of the SSHE, and $\omega$ is the angular speed of the rotor.

The velocities, pressures, transverse volume fluxes (per unit length in the axial direction), temperatures and the axial volume flux are denoted by $u_{k}(x, y) \mathbf{i}+v_{k}(x, y) \mathbf{j}+$ $w_{k}(x, y) \mathbf{k}, P_{k}(x, y, z), Q_{k}, T_{k}(x, y)$ and $\mathcal{Q}$, respectively, where the subscripts $k=1,2,3$ denote quantities in each of the three regions. 
We non-dimensionalise and scale the variables appearing in the model according to

$$
\begin{gathered}
x=L x^{*}, \quad y=H y^{*}, \quad z=\frac{\mu_{0} U L}{G H^{2}} z^{*}, \quad h=H h^{*}, \quad l=L l^{*}, \\
u_{k}=U u_{k}^{*}, \quad v_{k}=\frac{U H}{L} v_{k}^{*}, \quad w_{k}=\frac{G H^{2}}{\mu_{0}} w_{k}^{*}, \\
P_{k}=\frac{\mu_{0} U L}{H^{2}} P_{k}^{*}, \quad Q_{k}=U H Q_{k}^{*}, \quad \mathcal{Q}=\frac{G H^{3} L}{\mu_{0}} \mathcal{Q}^{*}, \\
\mu=\mu_{0} \mu^{*}, \quad T_{k}=T_{0}+\left(T_{H}-T_{0}\right) T_{k}^{*},
\end{gathered}
$$

where $\mu_{0}=\mu\left(T_{0}\right)$. In the specific case of a linear blade, we scale the blade slope according to $\alpha=\epsilon \alpha^{*}=H \alpha^{*} / L$, where $0 \leqslant \alpha^{*} \leqslant 1$. In non-dimensional variables region 1 occupies $0 \leqslant x^{*}<1,0<y^{*}<h^{*}$, region 2 occupies $0 \leqslant x^{*}<1, h^{*}<y^{*}<1$, and region 3 occupies $1 \leqslant x^{*}<1+l^{*}, 0<y^{*}<1$. For simplicity, we immediately drop the superscript stars and henceforth all variables are non-dimensional and scaled unless stated otherwise.

Provided that both the reduced Reynolds number $\epsilon^{2} \operatorname{Re}=\epsilon^{2} \rho U L / \mu_{0}=o(1)$ and the reduced Péclet number $\epsilon^{2} \mathrm{Pe}=\epsilon^{2} \rho \mathrm{c}_{\mathrm{p}} U L / k=o(1)$, where $\rho, \mathrm{c}_{\mathrm{p}}$ and $k$ are the constant density, specific heat and thermal conductivity of the fluid, respectively, are small in the limit of small transverse aspect ratio, $\epsilon=H / L \rightarrow 0$, then at leading order the governing mass-conservation, Navier-Stokes and temperature equations yield

$$
\begin{gathered}
\frac{\partial u_{k}}{\partial x}+\frac{\partial v_{k}}{\partial y}=0, \\
\frac{\partial P_{k}}{\partial x}=\frac{\partial}{\partial y}\left(\mu \frac{\partial u_{k}}{\partial y}\right), \quad \frac{\partial P_{k}}{\partial y}=0, \quad \frac{\partial P_{k}}{\partial z}=-1=\frac{\partial}{\partial y}\left(\mu \frac{\partial w_{k}}{\partial y}\right), \\
\frac{\partial^{2} T_{k}}{\partial y^{2}}=0,
\end{gathered}
$$

subject to the boundary conditions

$$
\begin{aligned}
& \left.\begin{array}{lll}
u_{1}=1 & \text { on } & y=0 \\
T_{1}=0 & \text { on } & y=0 \\
u_{1}=0 & \text { on } & y=h^{-}
\end{array}\right\} \quad \text { for } \quad 0 \leqslant x<1 \\
& \left.\begin{array}{lll}
u_{2}=0 & \text { on } & y=h^{+} \\
u_{2}=0 & \text { on } & y=1 \\
T_{2}=1 & \text { on } & y=1
\end{array}\right\} \text { for } \quad 0 \leqslant x<1 \\
& \left.\begin{array}{lll}
u_{3}=1 & \text { on } & y=0 \\
T_{3}=0 & \text { on } & y=0 \\
u_{3}=0 & \text { on } & y=1 \\
T_{3}=1 & \text { on } & y=1
\end{array}\right\} \text { for } \quad 1 \leqslant x<1+l \\
& T_{1}=T_{2} \quad \text { and } \quad \frac{\partial T_{1}}{\partial y}=\frac{\partial T_{2}}{\partial y} \quad \text { on } \quad y=h \quad \text { for } \quad 0 \leqslant x<1, \\
& P_{2}(x=0)=P_{3}(x=1+l), \quad P_{1}(x=1)=P_{2}(x=1)=P_{3}(x=1),
\end{aligned}
$$

together with $v_{k}=w_{k}=0(k=1,2,3)$ on all solid boundaries. 


\subsection{General Solution of the Model}

It is easily shown that integrating (4) subject to the appropriate temperature boundary conditions contained in (5)-(8) gives the simple linear temperature distribution

$$
T_{k}=T=y,
$$

in each of the three regions, so that $\mu=\mu(y)$ everywhere. Equations (3b) and (3c) yield

$$
P_{k}=-z+p_{k}
$$

where $p_{k}=p_{k}(x)(k=1,2,3)$ is the contribution to the pressure due to the transverse flow, and hence the governing equations for $u_{k}$ and $w_{k}$ may be written as

$$
\frac{\partial}{\partial y}\left(\mu \frac{\partial u_{k}}{\partial y}\right)=\frac{\mathrm{d} p_{k}}{\mathrm{~d} x}, \quad \frac{\partial}{\partial y}\left(\mu \frac{\partial w_{k}}{\partial y}\right)=-1
$$

Finally, for simplicity in what follows, we introduce the constants $p_{0}$ and $p_{L}$ such that the continuity-of-pressure conditions (9) may be written as

$$
p_{2}(x=0)=p_{3}(x=1+l)=p_{0}, \quad p_{1}(x=1)=p_{2}(x=1)=p_{3}(x=1)=p_{L} .
$$

\subsubsection{Solution for the Transverse (i.e. the $x$ and $y$ ) Flow}

Solving (12a) for the $x$ velocities $u_{k}$ subject to the no-slip conditions on the lower boundaries of regions 1, 2 and 3 contained in (5)-(7) gives

$$
\begin{gathered}
u_{1}=1+p_{1 x} \int_{0}^{y} \frac{\tilde{y}}{\mu(\tilde{y})} \mathrm{d} \tilde{y}+A_{1}(x) \int_{0}^{y} \frac{1}{\mu(\tilde{y})} \mathrm{d} \tilde{y}, \\
u_{2}=p_{2 x} \int_{h}^{y} \frac{\tilde{y}}{\mu(\tilde{y})} \mathrm{d} \tilde{y}+A_{2}(x) \int_{h}^{y} \frac{1}{\mu(\tilde{y})} \mathrm{d} \tilde{y}, \\
u_{3}=1+p_{3 x} \int_{0}^{y} \frac{\tilde{y}}{\mu(\tilde{y})} \mathrm{d} \tilde{y}+A_{3}(x) \int_{0}^{y} \frac{1}{\mu(\tilde{y})} \mathrm{d} \tilde{y},
\end{gathered}
$$

where $A_{k}=A_{k}(x)(k=1,2,3)$ are arbitrary functions of $x$ and the $x$ subscripts denote $x$ derivatives. Imposing the no-slip conditions at the upper boundaries of regions 1,2 and 3 contained in (5)-(7) determines the $A_{k}$ to be

$$
A_{1}=-\frac{1+p_{1 x} J_{11}}{J_{10}}, \quad A_{2}=-\frac{p_{2 x} J_{21}}{J_{20}}, \quad A_{3}=-\frac{1+p_{3 x} J_{31}}{J_{30}},
$$

with $J_{k n}(k=1,2,3)$ denoting the integrals

$$
J_{1 n}=\int_{0}^{h} \frac{\tilde{y}^{n}}{\mu(\tilde{y})} \mathrm{d} \tilde{y}, \quad J_{2 n}=\int_{h}^{1} \frac{\tilde{y}^{n}}{\mu(\tilde{y})} \mathrm{d} \tilde{y}, \quad J_{3 n}=\int_{0}^{1} \frac{\tilde{y}^{n}}{\mu(\tilde{y})} \mathrm{d} \tilde{y} .
$$

Note that $J_{1 n}+J_{2 n}=J_{3 n}$, where $J_{1 n}$ and $J_{2 n}$ depend on $x$ (via the blade shape $h$ ), whereas $J_{3 n}$ is independent of $x$. The transverse volume fluxes $Q_{k}$ are then given by

$$
Q_{1}=\int_{0}^{h} u_{1} \mathrm{~d} y, \quad Q_{2}=\int_{h}^{1} u_{2} \mathrm{~d} y, \quad Q_{3}=\int_{0}^{1} u_{3} \mathrm{~d} y,
$$


and hence

$$
\begin{gathered}
Q_{1}=h+p_{1 x} \int_{0}^{h} \frac{\tilde{y}(h-\tilde{y})}{\mu(\tilde{y})} \mathrm{d} \tilde{y}+A_{1} \int_{0}^{h} \frac{h-\tilde{y}}{\mu(\tilde{y})} \mathrm{d} \tilde{y}=\frac{J_{11}+p_{1 x}\left(J_{11}^{2}-J_{10} J_{12}\right)}{J_{10}}, \\
Q_{2}=p_{2 x} \int_{h}^{1} \frac{\tilde{y}(1-\tilde{y})}{\mu(\tilde{y})} \mathrm{d} \tilde{y}+A_{2} \int_{h}^{1} \frac{1-\tilde{y}}{\mu(\tilde{y})} \mathrm{d} \tilde{y}=\frac{p_{2 x}\left(J_{21}^{2}-J_{20} J_{22}\right)}{J_{20}}, \\
Q_{3}=1+p_{3 x} \int_{0}^{1} \frac{\tilde{y}(1-\tilde{y})}{\mu(\tilde{y})} \mathrm{d} \tilde{y}+A_{3} \int_{0}^{1} \frac{1-\tilde{y}}{\mu(\tilde{y})} \mathrm{d} \tilde{y}=\frac{J_{31}+p_{3 x}\left(J_{31}^{2}-J_{30} J_{32}\right)}{J_{30}} .
\end{gathered}
$$

Since the flow is steady the $Q_{k}$ are independent of $x$. Since the blade makes contact with the lower wall, the transverse volume flux in region 1 must be zero, i.e. $Q_{1}=0$. Moreover, global conservation of mass then implies that the transverse volume fluxes in regions 2 and 3 must be equal, i.e. $Q_{2}=Q_{3}=Q$, say. Equations (20)-(22) may then be rearranged to obtain expressions for the pressure gradients, namely

$$
p_{1 x}=-\frac{J_{11}}{J_{11}^{2}-J_{10} J_{12}}, \quad p_{2 x}=\frac{Q J_{20}}{J_{21}^{2}-J_{20} J_{22}}, \quad p_{3 x}=\frac{Q J_{30}-J_{31}}{J_{31}^{2}-J_{30} J_{32}} .
$$

Note that $p_{1 x}$ and $p_{2 x}$ are functions of $x$ (via the blade shape $h$ ), whereas $p_{3 x}$ is independent of $x$. Finally, using the continuity-of-pressure conditions (13) we may integrate the expressions for the pressure gradients $p_{2 x}$ and $p_{3 x}$ given in (23b) and (23c) to yield two expressions for the pressure difference $p_{0}-p_{L}$, namely

$$
p_{0}-p_{L}=-\int_{0}^{1} \frac{Q J_{20}}{J_{21}^{2}-J_{20} J_{22}} \mathrm{~d} x=\frac{l\left(Q J_{30}-J_{31}\right)}{J_{31}^{2}-J_{30} J_{32}},
$$

which yield the transverse volume flux

$$
Q=\frac{l J_{31}}{l J_{30}+\left(J_{31}^{2}-J_{30} J_{32}\right) \int_{0}^{1} \frac{J_{20}}{J_{21}^{2}-J_{20} J_{22}} \mathrm{~d} x} .
$$

The final solutions for $u_{k}$ are obtained by substituting the expressions for the $p_{k x}$ given in (23) with the value of $Q$ given by (25) into (14)-(16) using (17).

Once the solutions for $u_{k}$ are known, the corresponding solutions for the $y$ velocities $v_{k}$ may be obtained if required from (2) subject to the no-penetration conditions $v_{k}=0$ on all solid boundaries. These solutions are omitted for brevity.

\subsubsection{Solution for the Axial (i.e. the $z$ ) Flow}

Solving $(12 \mathrm{~b})$ for the axial (i.e. the $z$ ) velocities $w_{k}$ subject to the no-slip conditions $w_{k}=0$ on all solid boundaries gives

$$
\begin{aligned}
& w_{1}=\frac{J_{11}}{J_{10}} \int_{0}^{y} \frac{1}{\mu(\tilde{y})} \mathrm{d} \tilde{y}-\int_{0}^{y} \frac{\tilde{y}}{\mu(\tilde{y})} \mathrm{d} \tilde{y}, \\
& w_{2}=\frac{J_{21}}{J_{20}} \int_{h}^{y} \frac{1}{\mu(\tilde{y})} \mathrm{d} \tilde{y}-\int_{h}^{y} \frac{\tilde{y}}{\mu(\tilde{y})} \mathrm{d} \tilde{y}, \\
& w_{3}=\frac{J_{31}}{J_{30}} \int_{0}^{y} \frac{1}{\mu(\tilde{y})} \mathrm{d} \tilde{y}-\int_{0}^{y} \frac{\tilde{y}}{\mu(\tilde{y})} \mathrm{d} \tilde{y} .
\end{aligned}
$$


The axial volume flux across the section $0 \leqslant x<1+l$ and $0<y<1$ is then given by

$$
\mathcal{Q}=\int_{0}^{1} \int_{0}^{h} w_{1} \mathrm{~d} y \mathrm{~d} x+\int_{0}^{1} \int_{h}^{1} w_{2} \mathrm{~d} y \mathrm{~d} x+\int_{1}^{1+l} \int_{0}^{1} w_{3} \mathrm{~d} y \mathrm{~d} x
$$

which reduces to

$$
\mathcal{Q}=\int_{0}^{1} \frac{J_{10} J_{20}\left(J_{12}+J_{22}\right)-J_{10} J_{21}^{2}-J_{20} J_{11}^{2}}{J_{10} J_{20}} \mathrm{~d} x+\frac{l\left(J_{30} J_{32}-J_{31}^{2}\right)}{J_{30}} .
$$

\subsection{Exponential Dependence of Viscosity on Temperature}

In order to proceed much further it is necessary to specify the functional form of the dependence of viscosity $\mu$ on temperature $T$, and hence for the remainder of the present work we will focus on the specific case of an exponential dependence of viscosity on temperature given (in dimensional variables) by

$$
\mu(T)=\mu_{0} \exp \left[\frac{-\lambda\left(T-T_{0}\right)}{\mu_{0}}\right]
$$

where $\mu_{0}=\mu\left(T_{0}\right)$ and the sign of the constant $\lambda\left(=-\mathrm{d} \mu /\left.\mathrm{d} T\right|_{T=T_{0}}\right)$ determines whether the fluid is heat-thinning $(\lambda>0)$ or heat-thickening $(\lambda<0)$. Making use of (1) and recalling from (10) that $T_{k}=T=y$ we may express (31) in non-dimensional form as

$$
\mu=\mathrm{e}^{-V T}=\mathrm{e}^{-V y}
$$

where

$$
V=\frac{\lambda\left(T_{H}-T_{0}\right)}{\mu_{0}}
$$

is a non-dimensional measure of the variation of viscosity with temperature known as the thermoviscosity number (see, for example, Wilson and Duffy [22]).

Substituting (32) into (14)-(16) using (17) yields

$$
\begin{gathered}
u_{1}=\frac{p_{1 x}\left[h \mathrm{e}^{V h}-y \mathrm{e}^{V y}-(h-y) \mathrm{e}^{V(y+h)}\right]+V\left(\mathrm{e}^{V h}-\mathrm{e}^{V y}\right)}{V\left(\mathrm{e}^{V h}-1\right)}, \\
u_{2}=\frac{p_{2 x}\left[(1-h) \mathrm{e}^{V(h+1)}-(1-y) \mathrm{e}^{V(y+1)}-(y-h) \mathrm{e}^{V(y+h)}\right]}{V\left(\mathrm{e}^{V}-\mathrm{e}^{V h}\right)}, \\
u_{3}=\frac{p_{3 x}\left[\mathrm{e}^{V}-y \mathrm{e}^{V y}-(1-y) \mathrm{e}^{V(y+1)}\right]+V\left(\mathrm{e}^{V}-\mathrm{e}^{V y}\right)}{V\left(\mathrm{e}^{V}-1\right)} .
\end{gathered}
$$

Plots of the $u_{k}$ will be given in Sec. 5 for the most realistic case of a linear blade. The transverse volume fluxes (20)-(22) are then given in terms of the pressure gradients by

$$
\begin{gathered}
Q_{1}=\frac{p_{1 x}\left[V^{2} h^{2} \mathrm{e}^{V h}-\left(\mathrm{e}^{V h}-1\right)^{2}\right]+V^{2}\left[(V h-1) \mathrm{e}^{V h}+1\right]}{V^{3}\left(\mathrm{e}^{V h}-1\right)}, \\
Q_{2}=\frac{p_{2 x}\left[V^{2}(1-h)^{2} \mathrm{e}^{V(h+1)}-\left(\mathrm{e}^{V h}-\mathrm{e}^{V}\right)^{2}\right]}{V^{3}\left(\mathrm{e}^{V}-\mathrm{e}^{V h}\right)},
\end{gathered}
$$




$$
Q_{3}=\frac{p_{3 x}\left[V^{2} \mathrm{e}^{V}-\left(\mathrm{e}^{V}-1\right)^{2}\right]+V^{2}\left[(V-1) \mathrm{e}^{V}+1\right]}{V^{3}\left(\mathrm{e}^{V}-1\right)} .
$$

From (23a) (equivalent to setting $Q_{1}=0$ in (37)) the pressure gradient in region 1 is given by

$$
p_{1 x}=\frac{V^{2}\left[(V h-1) \mathrm{e}^{V h}+1\right]}{\left(\mathrm{e}^{V h}-1\right)^{2}-V^{2} h^{2} \mathrm{e}^{V h}},
$$

which may be substituted into (34) to give

$$
u_{1}=\frac{V\left[(V h-1) \mathrm{e}^{V h}+1\right]\left[h \mathrm{e}^{V h}-y \mathrm{e}^{V y}-(h-y) \mathrm{e}^{V(y+h)}\right]+\left[\left(\mathrm{e}^{V h}-1\right)^{2}-V^{2} h^{2} \mathrm{e}^{V h}\right]\left(\mathrm{e}^{V h}-\mathrm{e}^{V y}\right)}{\left[\left(\mathrm{e}^{V h}-1\right)^{2}-V^{2} h^{2} \mathrm{e}^{V h}\right]\left(\mathrm{e}^{V h}-1\right)} .
$$

By specifying a specific form for the blade shape $h$ it is, in principle, possible to obtain an explicit expression for the pressure in region 1 by direct integration of (40). It is worth noting at this point that the pressure gradient (40) has a singularity at $x=0$ where $p_{1 x} \rightarrow \infty$ as $x \rightarrow 0^{+}$. In particular, if the blade is locally linear near $x=0$ like $h \sim \alpha x$ then we may show that

$$
\begin{aligned}
& p_{1 x} \sim \frac{6}{\alpha^{2} x^{2}}-\frac{2 V}{\alpha x} \rightarrow \infty, \quad p_{1} \sim-\frac{6}{\alpha^{2} x}-\frac{2 V \ln x}{\alpha} \rightarrow-\infty \\
& \left.\frac{\partial u_{1}}{\partial y}\right|_{y=0} \sim-\frac{4}{\alpha x}+V \rightarrow-\infty,\left.\quad \frac{\partial u_{1}}{\partial y}\right|_{y=h} \sim \frac{2}{\alpha x}+V \rightarrow \infty
\end{aligned}
$$

as $x \rightarrow 0^{+}$, meaning that the forces on the blade and the lower wall $y=0$, along with the moment on the blade about the pivot, are all infinite, implying that an infinite amount of power would be required to rotate the rotor. This issue is discussed in detail by Duffy et al. [17], who show that the singularities can be alleviated in a number of physically plausible ways by incorporating additional physical effects, such as shear-thinning fluid behaviour, slip at the boundaries or cavitation, into the model, and hence we do not need to pursue it further here.

From (23b) and (23c) (equivalent to setting $Q_{2}=Q_{3}=Q$ in (38) and (39)) the pressure gradients in regions 2 and 3 are given by

$$
\begin{gathered}
p_{2 x}=\frac{Q V^{3}\left(\mathrm{e}^{V}-\mathrm{e}^{V h}\right)}{V^{2}(1-h)^{2} \mathrm{e}^{V(h+1)}-\left(\mathrm{e}^{V}-\mathrm{e}^{V h}\right)^{2}}, \\
p_{3 x}=\frac{V^{2}\left[Q V\left(\mathrm{e}^{V}-1\right)-(V-1) \mathrm{e}^{V}-1\right]}{V^{2} \mathrm{e}^{V}-\left(\mathrm{e}^{V}-1\right)^{2}},
\end{gathered}
$$

and (24) yields two expressions for the pressure difference $p_{0}-p_{L}$, namely

$$
p_{0}-p_{L}=-Q V^{3} I=\frac{l V^{2}\left[Q V\left(\mathrm{e}^{V}-1\right)-(V-1) \mathrm{e}^{V}-1\right]}{V^{2} \mathrm{e}^{V}-\left(\mathrm{e}^{V}-1\right)^{2}},
$$

where the integral $I$ is defined as

$$
I=\int_{0}^{1} \frac{\mathrm{e}^{V}-\mathrm{e}^{V h}}{V^{2}(1-h)^{2} \mathrm{e}^{V(h+1)}-\left(\mathrm{e}^{V}-\mathrm{e}^{V h}\right)^{2}} \mathrm{~d} x .
$$

From (25) the transverse volume flux is given by

$$
Q=\frac{l\left[(V-1) \mathrm{e}^{V}+1\right]}{V\left\{I\left[V^{2} \mathrm{e}^{V}-\left(\mathrm{e}^{V}-1\right)^{2}\right]+l\left(\mathrm{e}^{V}-1\right)\right\}} .
$$


By examining each of the terms in (47) it is relatively straightforward to verify that $Q \geqslant 0$ and $Q<1$ for any blade shape $h$ and all values of $l(\geqslant 0)$ and $V$, with $Q \rightarrow 1$ as $l \rightarrow \infty$ and $V \rightarrow \infty$, i.e. that the transverse volume flux lies in the range $0 \leqslant Q<1$. Note that in the special case $V=0$ Duffy et al. [17] showed that the more restrictive condition $0 \leqslant Q<1 / 2$ holds.

Substituting (32) into (26)-(28) yields

$$
\begin{gathered}
w_{1}=\frac{(h-y) \mathrm{e}^{V(y+h)}+y \mathrm{e}^{V y}-h \mathrm{e}^{V h}}{V\left(\mathrm{e}^{V h}-1\right)}, \\
w_{2}=\frac{(y-h) \mathrm{e}^{V(y+h)}+(1-y) \mathrm{e}^{V(y+1)}-(1-h) \mathrm{e}^{V(h+1)}}{V\left(\mathrm{e}^{V}-\mathrm{e}^{V h}\right)}, \\
w_{3}=\frac{(1-y) \mathrm{e}^{V(y+1)}+y \mathrm{e}^{V y}-\mathrm{e}^{V}}{V\left(\mathrm{e}^{V}-1\right)},
\end{gathered}
$$

which satisfy $w_{k} \geqslant 0$ in the appropriate regions. Plots of the $w_{k}$ will be given in Sec. 5 for the most realistic case of a linear blade. From (30) the axial volume flux across the section $0 \leqslant x<1+l$ and $0<y<1$ is given by

$$
\begin{gathered}
\mathcal{Q}=\frac{1}{V^{3}} \int_{0}^{1}\left\{\frac{\left(\mathrm{e}^{V h}-1\right)^{2}-V^{2} h^{2} \mathrm{e}^{V h}}{\mathrm{e}^{V h}-1}+\frac{\left(\mathrm{e}^{V}-\mathrm{e}^{V h}\right)^{2}-V^{2}(1-h)^{2} \mathrm{e}^{V(h+1)}}{\mathrm{e}^{V}-\mathrm{e}^{V h}}\right\} \mathrm{d} x \\
+\frac{l\left[\left(\mathrm{e}^{V}-1\right)^{2}-V^{2} \mathrm{e}^{V}\right]}{V^{3}\left(\mathrm{e}^{V}-1\right)} .
\end{gathered}
$$

\section{Special Limiting Cases of the Geometry}

The general solutions described in Sec. 2 above are valid for any blade shape $y=h(x)$ satisfying $h(0)=0,0<h(x)<1$ for $0<x<1$ and $0<h(1) \leqslant 1$ and for all values of the blade separation length $l(\geqslant 0)$ and the thermoviscosity number $V$. In Sec. 4 we will analyse the asymptotic behaviour of the solutions in the limits of weak and strong thermoviscosity; however before doing this it is of interest to study the behaviour in various special limiting cases of the geometry.

\subsection{The Special Case $h(x) \equiv 0$}

In the special case $h(x) \equiv 0$ the blade lies flat against the moving lower wall, removing region 1 from the problem and shielding region 2 from the direct effect of the lower wall (although it still feels an indirect effect via the flow generated in region 3 ). Setting $h(x) \equiv 0$ in (46) yields

$$
I=\frac{\mathrm{e}^{V}-1}{V^{2} \mathrm{e}^{V}-\left(\mathrm{e}^{V}-1\right)^{2}}
$$

and hence from (47) the transverse volume flux is given by

$$
Q=\frac{l\left[(V-1) \mathrm{e}^{V}+1\right]}{V(l+1)\left(\mathrm{e}^{V}-1\right)} .
$$

Substituting (53) into (43) and (44) yields the pressure gradients in regions 2 and 3, namely

$$
p_{2 x}=-\frac{l V^{2}\left[(V-1) \mathrm{e}^{V}+1\right]}{(l+1)\left[\left(\mathrm{e}^{V}-1\right)^{2}-V^{2} \mathrm{e}^{V}\right]}
$$




$$
p_{3 x}=\frac{V^{2}\left[(V-1) \mathrm{e}^{V}+1\right]}{(l+1)\left[\left(\mathrm{e}^{V}-1\right)^{2}-V^{2} \mathrm{e}^{V}\right]},
$$

and substituting (53) into (35) and (36) yields the $x$ velocities in regions 2 and 3 (omitted for brevity). Substituting $h(x) \equiv 0$ into (49) yields the axial velocity

$$
w_{2}=\frac{\left[(1-y) \mathrm{e}^{V}+y\right] \mathrm{e}^{V y}-\mathrm{e}^{V}}{V\left(\mathrm{e}^{V}-1\right)},
$$

(with $w_{3}$ still given by (50)), and substituting into (51) yields the axial volume flux

$$
\mathcal{Q}=\frac{(l+1)\left[\left(\mathrm{e}^{V}-1\right)^{2}-V^{2} \mathrm{e}^{V}\right]}{V^{3}\left(\mathrm{e}^{V}-1\right)} .
$$

Note that in this case, but not in general, the transverse flow in region 2 is rectilinear (like it always is in region 3 ) and hence the pressure gradient in region 2 is independent of $x$ (like it always is in region 3 ).

\subsection{The Special Case $h(1)=1$}

In the special case $h(1)=1$ the right-hand end of the blade makes contact with the stationary upper wall and completely blocks the channel. Since the fluid cannot pass over the blade, the transverse volume flux in regions 2 and 3 must be zero (like that in region 1) so that $Q_{2}=Q_{3}=Q=0$. The form of the solution in region 1 is unaffected, but setting $Q=0$ in (43) and (44) the pressure gradients in regions 2 and 3 are given by

$$
p_{2 x}=0, \quad p_{3 x}=-\frac{V^{2}\left[(V-1) \mathrm{e}^{V}+1\right]}{V^{2} \mathrm{e}^{V}-\left(\mathrm{e}^{V}-1\right)^{2}} .
$$

Substituting (58) into (35) and (36) yields $u_{2}=0$ and

$$
u_{3}=\frac{\left\{(V y+1)\left(\mathrm{e}^{V}-1\right)+V[V(1-y)-1] \mathrm{e}^{V}\right\} \mathrm{e}^{V y}-\left(\mathrm{e}^{V}-V-1\right) \mathrm{e}^{V}}{V^{2} \mathrm{e}^{V}-\left(\mathrm{e}^{V}-1\right)^{2}}
$$

while the axial velocities $w_{k}$ and the axial volume flux $\mathcal{Q}$ are still given by (48)-(51). Note that since region 2 is entirely shielded from the effect of the moving lower wall, there is no transverse flow in this region in this case.

\subsection{The Special Case $l=0$}

In the special case $l=0$ there is no separation between adjacent blades, eliminating region 3 from the problem. Since region 2 is again completely shielded from the moving lower wall, the transverse volume flux in region 2 is zero, i.e. $Q_{2}=Q=0$. The form of the solution in region 1 is again unaffected, but (35) and (43) yield $p_{2 x}=0$ and $u_{2}=0$ in region 2. The axial velocities $w_{1}$ and $w_{2}$ are still given by (48) and (49), while the axial volume flux $\mathcal{Q}$ is given by (51) with $l=0$. Note that there is again no transverse flow in region 2 in this case. 


\subsection{The Limit $l \rightarrow \infty$}

In the limit $l \rightarrow \infty$ the separation between adjacent blades becomes infinitely large and the problem becomes that of a single blade in an infinitely long channel. From (47) the leading order behaviour of the transverse volume flux in the limit $l \rightarrow \infty$ is independent of the shape of the blade, specifically

$$
Q \sim \frac{(V-1) \mathrm{e}^{V}+1}{V\left(\mathrm{e}^{V}-1\right)}=O(1)
$$

The form of the solution in region 1 is again unaffected, but substituting (60) into (35) and (43) yields

$$
p_{2 x} \sim \frac{V^{2}\left[(V-1) \mathrm{e}^{V}+1\right]\left(\mathrm{e}^{V}-\mathrm{e}^{V h}\right)}{\left(\mathrm{e}^{V}-1\right)\left[V^{2}(1-h)^{2} \mathrm{e}^{V(h+1)}-\left(\mathrm{e}^{V}-\mathrm{e}^{V h}\right)^{2}\right]}
$$

and

$$
u_{2} \sim \frac{V\left[(V-1) \mathrm{e}^{V}+1\right]\left[(1-h) \mathrm{e}^{V(h+1)}-(1-y) \mathrm{e}^{V(y+1)}-(y-h) \mathrm{e}^{V(y+h)}\right]}{\left(\mathrm{e}^{V}-1\right)\left[V^{2}(1-h)^{2} \mathrm{e}^{V(h+1)}-\left(\mathrm{e}^{V}-\mathrm{e}^{V h}\right)^{2}\right]} .
$$

Substituting (60) into (36) and (44) yields $p_{3 x}=o(1)$ and

$$
u_{3} \sim \frac{\mathrm{e}^{V}-\mathrm{e}^{V y}}{\mathrm{e}^{V}-1} .
$$

In fact, expanding (47) to next order reveals that

$$
p_{3 x} \sim-\frac{I V^{2}\left[(V-1) \mathrm{e}^{V}+1\right]}{l\left(\mathrm{e}^{V}-1\right)}=O\left(l^{-1}\right) \rightarrow 0 .
$$

The axial velocities $w_{k}$ are still given by (48)-(50), while from (51) the leading order axial volume flux is independent of the shape of the blade and is given by

$$
\mathcal{Q} \sim \frac{l\left[\left(\mathrm{e}^{V}-1\right)^{2}-V^{2} \mathrm{e}^{V}\right]}{V^{3}\left(\mathrm{e}^{V}-1\right)}=O(l) \rightarrow \infty .
$$

\section{The Limits of Weak and Strong Thermoviscosity Effects}

In Secs 2 and 3 we derived and analysed explicit solutions for the heat and fluid flow in the present mathematical model of a narrow-gap SSHE. Unfortunately, in general, several of the expressions are rather cumbersome and some or all of the integrals involved may need to be evaluated numerically, making it difficult to understand fully the effect of the governing parameters on the behaviour of the solutions. In order to help to elucidate the effect of thermoviscosity on the solutions (and in order to provide useful analytical checks on the numerical results presented in Sec. 5), in this section we describe the behaviour of the solutions in the asymptotic limits of weak and strong thermoviscosity. Small thermoviscosity (i.e. the limit $V \rightarrow 0$ ) corresponds to a weak heating or cooling of the rotor relative to the stator and/or a weak dependence of viscosity on temperature. 
Large positive thermoviscosity (i.e. the limit $V \rightarrow \infty$ ) corresponds to a strong heating of the rotor relative to the stator for a heat thinning fluid or a strong cooling of the rotor relative to the stator for a heat thickening fluid and/or a strongly heat thinning fluid when the rotor is heated relative to the stator or a strongly heat thickening fluid when the rotor is cooled relative to the stator, with the corresponding meanings for large negative thermoviscosity (i.e. the limit $V \rightarrow-\infty$ ).

\subsection{Small Thermoviscosity $V \rightarrow 0$}

In the special case $V=0$ (corresponding to the rotor and the stator being at the same temperature and/or no dependence of viscosity on temperature) we recover the appropriate versions of the isothermal solutions obtained by Duffy et al. [17]. In this subsection we investigate the effect of weak thermoviscosity by obtaining the regular small $V$ expansions about these solutions.

Expanding (40) and (41) for small $V$ yields

$$
p_{1 x}=\frac{6}{h^{2}}-\frac{2}{h} V+O\left(V^{2}\right)
$$

and

$$
u_{1}=\frac{(h-3 y)(h-y)}{h^{2}}+\frac{y(h-2 y)(h-y)}{h^{2}} V+O\left(V^{2}\right)
$$

and hence

$$
p_{1}-p_{L}=\int_{1}^{x} \frac{6}{h(\tilde{x})^{2}} \mathrm{~d} \tilde{x}-\left(\int_{1}^{x} \frac{2}{h(\tilde{x})} \mathrm{d} \tilde{x}\right) V+O\left(V^{2}\right) .
$$

Expanding (43) and (44) gives

$$
\begin{gathered}
p_{2 x}=-\frac{12 Q_{0}}{(1-h)^{3}}+\frac{6\left[Q_{0}(h+1)-2 Q_{1}\right]}{(1-h)^{3}} V+O\left(V^{2}\right), \\
p_{3 x}=6\left(1-2 Q_{0}\right)+2\left(3 Q_{0}-6 Q_{1}-1\right) V+O\left(V^{2}\right),
\end{gathered}
$$

where we have written $Q=Q_{0}+Q_{1} V+O\left(V^{2}\right)$, and expanding (45) yields two expressions for the pressure difference $p_{0}-p_{L}$, namely

$$
\begin{gathered}
p_{0}-p_{L}=12 Q_{0} I_{0}-6\left[Q_{0} I_{1}+\left(Q_{0}-2 Q_{1}\right) I_{0}\right] V+O\left(V^{2}\right), \\
p_{0}-p_{L}=6 l\left(1-2 Q_{0}\right)+2 l\left(3 Q_{0}-6 Q_{1}-1\right) V+O\left(V^{2}\right),
\end{gathered}
$$

where $I_{n}$ denotes the integral

$$
I_{n}=\int_{0}^{1} \frac{h^{n}}{(1-h)^{3}} \mathrm{~d} x
$$

Expanding (47) reveals that the transverse volume flux is given by

$$
Q=\frac{l}{2\left(l+I_{0}\right)}+\frac{l\left(l+I_{0}+3 I_{1}\right)}{12\left(l+I_{0}\right)^{2}} V+O\left(V^{2}\right) .
$$

Substituting (74) into (69) and (70) and integrating yields

$$
p_{2}-p_{L}=-\frac{6 l K_{0}}{l+I_{0}}+\frac{l\left[3\left(l+I_{0}\right) K_{1}+\left(2 l+2 I_{0}-3 I_{1}\right) K_{0}\right]}{\left(l+I_{0}\right)^{2}} V+O\left(V^{2}\right),
$$




$$
p_{3}-p_{L}=\frac{6 I_{0}(x-1)}{l+I_{0}}-\frac{\left[l\left(2 I_{0}+3 I_{1}\right)+2 I_{0}^{2}\right](x-1)}{\left(l+I_{0}\right)^{2}} V+O\left(V^{2}\right)
$$

where $K_{n}$ denotes the integral

$$
K_{n}=\int_{1}^{x} \frac{h(\tilde{x})^{n}}{[1-h(\tilde{x})]^{3}} \mathrm{~d} \tilde{x}
$$

Substituting (75) and (76) into (35) and (36) yields

$$
\begin{gathered}
u_{2}=\frac{3 l(y-h)(1-y)}{\left(l+I_{0}\right)(1-h)^{3}}-\frac{l\left[\left(l+I_{0}\right)(2 h-4 y+1)-3 I_{1}\right](1-y)(y-h)}{2\left(l+I_{0}\right)^{2}(1-h)^{3}} V+O\left(V^{2}\right), \\
u_{3}=\frac{\left[l+I_{0}-3 y I_{0}\right](1-y)}{l+I_{0}}+\frac{\left[l^{2}+\left(3 I_{0}-4 y I_{0}+3 I_{1}\right) l+2 I_{0}^{2}(1-2 y)\right] y(1-y)}{2\left(l+I_{0}\right)^{2}} V+O\left(V^{2}\right) .
\end{gathered}
$$

Expanding (48)-(50) for small $V$ it is straightforward to show that

$$
\begin{gathered}
w_{1}=\frac{y(h-y)}{2}+\frac{y(h-y)(4 y+h)}{12} V+O\left(V^{2}\right), \\
w_{2}=\frac{(1-y)(y-h)}{2}+\frac{(1-y)(y-h)(4 y+h+1)}{12} V+O\left(V^{2}\right), \\
w_{3}=\frac{y(1-y)}{2}+\frac{y(1-y)(4 y+1)}{12} V+O\left(V^{2}\right),
\end{gathered}
$$

and hence from (51) that the axial volume flux is given by

$$
\mathcal{Q}=\frac{1}{12}\left[1+\int_{0}^{1}\left(3 h^{2}-3 h+1\right) \mathrm{d} x\right]+\frac{1}{24}\left[l+\int_{0}^{1}\left(2 h^{3}-2 h+1\right) \mathrm{d} x\right] V+O\left(V^{2}\right)
$$

\subsection{Large Positive Thermoviscosity $V \rightarrow \infty$}

At leading order in the limit of large positive thermoviscosity, $V \rightarrow \infty$, equation (40) yields

$$
p_{1 x} \sim V^{3} h \mathrm{e}^{-V h},
$$

and hence the leading order pressure in region 1 is given by

$$
p_{1}-p_{L} \sim V^{3} \int_{1}^{x} h(\tilde{x}) \mathrm{e}^{-V h(\tilde{x})} \mathrm{d} \tilde{x}
$$

while from (41) the $x$ velocity in region 1 is given by

$$
u_{1} \sim 1-[V(V h-1)(h-y)+1] \mathrm{e}^{-V(h-y)} .
$$

From (47) the leading order transverse volume flux is given by

$$
Q \sim \frac{l}{l+1}=O(1)
$$

Expanding (43) and (44) yields

$$
p_{2 x} \sim-\frac{l V^{3}}{(l+1) \mathrm{e}^{V}}, \quad p_{3 x} \sim \frac{V^{3}}{(l+1) \mathrm{e}^{V}},
$$


and hence the leading order pressures in regions 2 and 3 are

$$
p_{2}-p_{L} \sim \frac{l V^{3}(1-x)}{(l+1) \mathrm{e}^{V}}, \quad p_{3}-p_{L} \sim \frac{V^{3}(x-1)}{(l+1) \mathrm{e}^{V}},
$$

while from (35) and (36) the $x$ velocities in regions 2 and 3 are given by

$$
u_{2} \sim \frac{l V^{2}(1-y) \mathrm{e}^{-V(1-y)}}{l+1}, \quad u_{3} \sim 1-\frac{[l+1+V(V-1)(1-y)] \mathrm{e}^{-V(1-y)}}{l+1} .
$$

Expanding (48)-(50) yields the axial velocities

$$
w_{1} \sim \frac{(h-y) \mathrm{e}^{V y}}{V}, \quad w_{2} \sim \frac{(1-y) \mathrm{e}^{V y}}{V}, \quad w_{3} \sim \frac{(1-y) \mathrm{e}^{V y}}{V},
$$

and hence from (51) the leading order axial volume flux is

$$
\mathcal{Q} \sim \frac{(l+1) \mathrm{e}^{V}}{V^{3}}=O\left(\frac{\mathrm{e}^{V}}{V^{3}}\right) \rightarrow \infty .
$$

Figure 3 shows sketches of the asymptotic solutions for the $x$ velocities in each of the three regions given by (86) and (90) highlighting the magnitude of the velocity in the different parts of the flow. In all three regions the viscosity is an exponentially decreasing function of $y$, and hence in each region the bulk of the fluid has an exponentially larger viscosity than that near its upper boundary. Hence in each region the bulk of the fluid moves as a rigid body with the velocity of its lower boundary (namely $u_{1}=1$, $u_{2}=0$ and $u_{3}=1$ ) with an appropriate boundary layer just below its upper boundary. In region 1 the transverse volume flux is zero and there is a boundary layer of width $O\left(V^{-1}\right)$ just below the blade in which there is backwards flow (i.e. $u_{1}<0$ ) with velocity $O(V)$, resulting in a negative $O(1)$ volume flux in the boundary layer which is equal and opposite to the positive $O(1)$ volume flux in the bulk of the region. In regions 2 and 3 there are boundary layers of width $O\left(V^{-1}\right)$ just below the stationary upper wall in which there is forwards flow with velocity $O(V)$ in region 2 and backwards flow with velocity $O(V)$ in region 3, resulting in an $O(1)$ transverse volume flux.

Figure 4 shows the corresponding sketches of the asymptotic solutions for the axial (i.e. the $z$ ) velocities in each of the three regions given by (91). The axial velocities have rather similar behaviour to that of the $x$ velocities, namely that in each region the bulk of the fluid is stationary (namely $w_{1}=w_{2}=w_{3}=0$ ) with an appropriate boundary layer of width $O\left(V^{-1}\right)$ just below its upper boundary in which there is forwards flow with velocity $O\left(V^{-2} \mathrm{e}^{V h}\right)$ just below the blade in region 1 and forwards flow with velocity $O\left(V^{-2} \mathrm{e}^{V}\right)$ just below the stationary upper wall in regions 2 and 3 , resulting in a large $O\left(V^{-3} \mathrm{e}^{V}\right)$ axial volume flux.

In particular, Figs 3 and 4 show that in the limit of large positive thermoviscosity the fastest flow occurs in boundary layers where the viscosity is lowest in each region (i.e. just below the blade and just below the stationary upper wall) in which the $x$ velocity is $O(V)$ larger than the velocity of the moving lower wall driving the transverse flow, and in which all of the axial flow occurs. Note that since the $x$ and the axial velocities in the boundary layers grow unboundedly with $V$, hitherto neglected inertial effects will always eventually become significant in this limit. 


\subsection{Large Negative Thermoviscosity $V \rightarrow-\infty$}

At leading order in the limit of large negative thermoviscosity, $V \rightarrow-\infty$, equation (40) yields

$$
p_{1 x} \sim V^{2}
$$

and hence the leading order pressure in region 1 is given by

$$
p_{1}-p_{L} \sim-V^{2}(1-x),
$$

while from (41) the $x$ velocity in region 1 is given by

$$
u_{1} \sim(1-|V| y) \mathrm{e}^{-|V| y} .
$$

From (47) the leading order transverse volume flux is given by

$$
Q \sim \frac{l}{|V| \widetilde{I}}
$$

where $\widetilde{I}$ is the leading order term in the expansion of $I$ given by (46) as $V \rightarrow-\infty$, i.e.

$$
\widetilde{I}=\int_{0}^{1} \mathrm{e}^{|V| h} \mathrm{~d} x .
$$

Note that in the most realistic case of a linear blade $h=\alpha x$ we have $\widetilde{I} \sim \mathrm{e}^{\alpha|V|} / \alpha|V|$ and hence $Q \sim l \alpha \mathrm{e}^{-\alpha|V|}=O\left(\mathrm{e}^{-\alpha|V|}\right) \rightarrow 0^{+}$. Expanding (43) and (44) yields

$$
p_{2 x} \sim-\frac{l V^{2} \mathrm{e}^{|V| h}}{\widetilde{I}}, \quad p_{3 x} \sim V^{2}
$$

and hence the leading order pressures in regions 2 and 3 are

$$
p_{2}-p_{L} \sim-\frac{l V^{2}}{\widetilde{I}} \int_{1}^{x} \mathrm{e}^{|V| h(\tilde{x})} \mathrm{d} \tilde{x}, \quad p_{3}-p_{L} \sim V^{2}(x-1),
$$

while from (35) and (36) the $x$ velocities in regions 2 and 3 are given by

$$
u_{2} \sim \frac{l|V|(y-h) \mathrm{e}^{-|V|(y-h)}}{\widetilde{I}}, \quad u_{3} \sim(1-|V| y) \mathrm{e}^{-|V| y} .
$$

Expanding (48)-(50) yields the axial velocities

$$
w_{1} \sim \frac{y \mathrm{e}^{-|V| y}}{|V|}, \quad w_{2} \sim \frac{(y-h) \mathrm{e}^{-|V| y}}{|V|}, \quad w_{3} \sim \frac{y \mathrm{e}^{-|V| y}}{|V|},
$$

and hence from (51) the leading order axial volume flux is

$$
\mathcal{Q} \sim \frac{l+1}{|V|^{3}}=O\left(|V|^{-3}\right) \rightarrow 0^{+} .
$$

Figure 5 shows sketches of the asymptotic solutions for the $x$ velocities in each of the three regions given by (95) and (100) again highlighting the magnitude of the velocity in the different parts of the flow. In contrast to the situation discussed in Sec. 
4.2 , in all three regions the viscosity is an exponentially increasing function of $y$, and hence in each region the bulk of the fluid has an exponentially larger viscosity than that near its lower boundary. Hence in each region the bulk of the fluid is stationary (namely $u_{1}=u_{2}=u_{3}=0$ ) with an appropriate boundary layer just above its lower boundary. In region 1 the transverse volume flux is zero and there is a boundary layer of width $O\left(|V|^{-1}\right)$ just above the moving lower wall in which there is both forwards and backwards flow with velocity $O(1)$, resulting in zero net volume flux in the boundary layer. In regions 2 and 3 there are boundary layers of width $O\left(|V|^{-1}\right)$ just above the lower boundary in which there is forwards flow with velocity $O\left(\widetilde{I}^{-1}\right)$ just above the blade in region 2 and forwards and backwards flow with velocity $O(1)$ just above the moving lower wall in region 3 , resulting in an $O\left(|V|^{-1} \widetilde{I}^{-1}\right)$ transverse volume flux. Note that in this limit the maximum magnitudes of the backwards flows in regions 1 and 3 approach the same $O(1)$ values, namely $u_{1} \sim u_{3} \sim-\mathrm{e}^{-2}$.

Figure 6 shows the corresponding sketches of the asymptotic solutions for the axial (i.e. the $z$ ) velocities in each of the three regions given by (101). The axial velocities again have rather similar behaviour to that of the $x$ velocities, namely that in each region the bulk of the fluid is stationary (namely $w_{1}=w_{2}=w_{3}=0$ ) with an appropriate boundary layer of width $O\left(|V|^{-1}\right)$ just above its lower boundary in which there is forwards flow with velocity $O\left(V^{-2}\right)$ just above the moving lower wall in regions 1 and 3 and forwards flow with velocity $O\left(V^{-2} \mathrm{e}^{-|V| h}\right)$ just above the blade in region 2 , resulting in a small $O\left(|V|^{-3}\right)$ axial volume flux.

In particular, Figs 5 and 6 show that in the limit of large negative thermoviscosity all of the transverse and axial flow occurs in boundary layers where the viscosity is lowest in each region (i.e. just above the blade and just above the moving lower wall).

\section{General Results and Discussion}

In this section we describe the behaviour of the solutions for a fluid with an exponential dependence of viscosity on temperature described in Sec. 2.3 for a wide range of parameter values. Where appropriate, comparisons are made with the asymptotic solutions in the limits of weak and strong thermoviscosity described in Sec. 4.

In order to calculate the solutions we need to prescribe the blade shape and, as indicated earlier, we consider the most realistic case of a linear blade given by $h=\alpha x$, with slope $\alpha$ satisfying $0 \leqslant \alpha \leqslant 1$.

Figures $7-12$ show plots of the pressure differences $p_{k}-p_{L}$ as functions of $x$, the $x$ velocities $u_{k}$ as functions of $y$, and the axial velocities $w_{k}$ as functions of $y$, for a range of positive values of the thermoviscosity number $V$ (Figs 7, 9 and 11) and a range of negative values of $V$ (Figs 8, 10 and 12) in the case $\alpha=0.5$ and $l=1$. In all of these figures the solid curves are the exact solutions obtained in Sec. 2.3, and the dashed curves are the small $V$ asymptotic solutions described in Sec. 4.1 and the large positive and negative $V$ asymptotic solutions described in Secs 4.2 and 4.3, respectively.

When $V=0$ the viscosity is constant over the entire channel $(\mu \equiv 1)$ and we recover the appropriate versions of the isothermal solutions obtained by Duffy et al. [17] given in Sec. 4.1 in which both the $x$ and the axial velocities are parabolic in $y$.

As $V$ increases from zero the viscosity becomes more and more strongly decreasing with $y$ across the channel, and Figs 7, 9 and 11 show how the $x$ and the axial velocities become progressively more uniform in the bulk of the flow with thin boundary layers 
forming just below the blade and just below the stationary upper wall, until ultimately the large positive $V$ asymptotic solutions described in Sec. 4.2 are attained in the limit $V \rightarrow \infty$. Similarly, as $V$ decreases from zero the viscosity becomes more and more strongly increasing with $y$ across the channel, and Figs 8, 10 and 12 show how the $x$ and the axial velocities become progressively more uniform in the bulk of the flow with thin boundary layers forming just above the blade and just above the moving lower wall, until ultimately the large negative $V$ asymptotic solutions described in Sec. 4.3 are attained in the limit $V \rightarrow-\infty$. In particular, Figs $7-12$ show that the small $V$ solutions are in good agreement with the exact solutions for values as large as $|V|=1$, while the large positive $V$ solutions are in good agreement with the exact solutions for values as small as $V=25$ and the large negative $V$ solutions are in good agreement with the exact solutions for values as small in magnitude as $V=-25$, albeit with a non-uniformity in the solution for $p_{1}$ near $x=0$ due to the singularity discussed in Sec. 2.3 .

Figure 13 shows the transverse volume flux $Q$ given by (47) plotted as a function of $V$ for a range of values of $\alpha$ in the case $l=1$. In particular, Fig. 13(b) shows that the solutions are in good agreement with the asymptotic solutions in the limits $V \rightarrow 0$, $V \rightarrow \infty$ and $V \rightarrow-\infty$ given by (74), (87) and (96), respectively. Figure 13 shows that $Q$ is a monotonically increasing function of $V$ satisfying $Q=O\left(\mathrm{e}^{-\alpha|V|}\right) \rightarrow 0^{+}$as $V \rightarrow-\infty$ and $Q \sim l /(l+1)=O(1)$ as $V \rightarrow \infty$ for fixed $\alpha$, and a monotonically decreasing function of $\alpha$ for fixed $V$. Figure 14 shows the axial volume flux $\mathcal{Q}$ given by (51) plotted as a function of $V$ for a range of values of $\alpha$ in the case $l=1$. In particular, Fig. 14(b) shows that the solutions are in good agreement with the asymptotic solutions in the limits $V \rightarrow 0, V \rightarrow \infty$ and $V \rightarrow-\infty$ given by (83), (92) and (102), respectively. Figure 14 shows that $\mathcal{Q}$ is a monotonically increasing function of $V$ satisfying $\mathcal{Q}=O\left(|V|^{-3}\right) \rightarrow 0^{+}$ as $V \rightarrow-\infty$ and $\mathcal{Q}=O\left(V^{-3} \mathrm{e}^{V}\right) \rightarrow \infty$ as $V \rightarrow \infty$ for fixed $\alpha$. Figure 14 clearly shows that $\mathcal{Q}$ is relatively insensitive to the value of $\alpha$, but what is not so immediately apparent from Fig. 14 is that $\mathcal{Q}$ is not a monotonically decreasing function of $\alpha$ for fixed $V$. In the case $V=0$ Duffy et al. [17] showed that $\mathcal{Q}$ takes its minimum value when $\alpha=\alpha_{\mathrm{m}}(0)=3 / 4$. In general, the minimum value of $\mathcal{Q}$ occurs at $\alpha=\alpha_{\mathrm{m}}(V)$ where, for example, $\alpha_{\mathrm{m}}(-3) \simeq 0.61$ and $\alpha_{\mathrm{m}}(3) \simeq 0.85$.

Figure 15 shows the transverse volume flux $Q$ given by (47) plotted as a function of $V$ for a range of values of $l$ in the case $\alpha=0.5$. Figure 15 shows that $Q$ is a monotonically increasing function of $V$ satisfying $Q=O\left(\mathrm{e}^{-\alpha|V|}\right) \rightarrow 0^{+}$as $V \rightarrow-\infty$ and $Q \sim l /(l+1)=O(1)$ as $V \rightarrow \infty$ for fixed $l$, and a monotonically increasing function of $l$ for fixed $V$. Finally, Fig. 16 shows the axial volume flux $\mathcal{Q}$ given by (51) plotted as a function of $V$ for a range of values of $l$ in the case $\alpha=0.5$. Figure 16 shows that $\mathcal{Q}$ is a monotonically increasing function of $V$ satisfying $\mathcal{Q}=O\left(|V|^{-3}\right) \rightarrow 0^{+}$as $V \rightarrow-\infty$ and $\mathcal{Q}=O\left(V^{-3} \mathrm{e}^{V}\right) \rightarrow \infty$ as $V \rightarrow \infty$ for fixed $l$, and a linearly increasing function of $l$ for fixed $V$.

In particular, Figs 13 and 15 show that provided that the right-hand end of the blade is not too close to the stationary upper wall and that the blades are not too close together (i.e. provided that $\alpha$ is not too close to 1 and $l$ is not too small) then there is a fairly narrow range of values of $V$, roughly $-10 \lesssim V \lesssim 20$, over which the transverse volume flux varies rapidly with $V$. 


\section{Conclusions}

In the present work we investigated the steady non-isothermal flow of a Newtonian fluid with temperature-dependent viscosity in a narrow-gap SSHE when a constant temperature difference is imposed across the gap between the rotor and the stator. In Sec. 2 we formulated the mathematical model and obtained the exact analytical solutions for the heat and fluid flow of a fluid with a general dependence of viscosity on temperature for a general blade shape. These solutions were then presented for the specific case of an exponential dependence of viscosity on temperature. In Sec. 3 asymptotic methods were employed to investigate the behaviour of the solutions in several special limiting geometries, while in Sec. 4 they were used to investigate the behaviour of the solutions in the limits of weak and strong thermoviscosity. In Sec. 5 we presented results for the most realistic case of a linear blade which illustrated the effect of varying the thermoviscosity of the fluid and the geometry of the SSHE on the flow. In particular, we found that as $V$ increases from zero the viscosity becomes more and more strongly decreasing with $y$ across the channel, and the $x$ and the axial velocities become progressively more uniform in the bulk of the flow with boundary layers forming just below the blade and just below the stationary upper wall, until ultimately the large positive $V$ asymptotic solutions described in Sec. 4.2 are attained in the limit $V \rightarrow \infty$ in which the transverse volume flux is $O(1)$ and the axial volume flux is $O\left(V^{-3} \mathrm{e}^{V}\right)$. Similarly, as $V$ decreases from zero the viscosity becomes more and more strongly increasing with $y$ across the channel, and the $x$ and the axial velocities become progressively more uniform in the bulk of the flow with boundary layers forming just above the blade and just above the moving lower wall, until ultimately the large negative $V$ asymptotic solutions described in Sec. 4.3 are attained in the limit $V \rightarrow-\infty$ in which the transverse volume flux is $O\left(\mathrm{e}^{-\alpha|V|}\right)$ and the axial volume flux is $O\left(|V|^{-3}\right)$. Furthermore, we showed that the maximum possible value of the transverse volume flux $Q=1$ is attained in the limits $V \rightarrow \infty$ and $l \rightarrow \infty$, and that provided that $\alpha$ is not too close to 1 and $l$ is not too small then there is a fairly narrow range of values of $V$, roughly $-10 \lesssim V \lesssim 20$, over which $Q$ varies rapidly with $V$.

While the present work represents a significant advance on the isothermal analysis of Duffy et al. [17], there are evidently several ways in which the work could be further developed. In particular, it would be of some practical interest to develop the corresponding analysis for thermally insulating blades. Duffy et al. [17] calculated the possible equilibrium positions of the blades in the isothermal case, and it would also be of interest to investigate the influence of thermal effects on these results. Although not included in the present model, viscous dissipation may well be significant in a real SSHE and it would therefore be valuable to include it in future models. In practice there is almost certainly some leakage of fluid under the blades, and so it would be of considerable interest to develop a model for this leakage and its effect on the overall heat and fluid flow within an SSHE. In a similar vein, in practice both the blades and the stator are slowly worn away by the scraping action of the blades, and so periodically the SSHE has to be taken out of service while the blades are replaced and/or the stator is re-coated. It would therefore be of great practical value to develop mathematical models for blade wear which could predict the most cost-effective moment to replace the blades. These and other related open questions indicate that SSHEs will remain the subject of active scientific research for a considerable time to come. 


\section{Acknowledgements}

This work was supported by the United Kingdom Engineering and Physical Sciences Research Council (EPSRC) via the University of Strathclyde Collaborative Training Account (CTA). All four authors wish to thank Professor Alistair Fitt (School of Mathematics, University of Southampton) for his encouragement and support throughout the course of the present work. On a personal note, the second author (SKW) welcomes the opportunity afforded by this special issue of the Journal of Engineering Mathematics to put on record his deep gratitude to Professor Norman Riley (School of Mathematics, University of East Anglia) for the unfailingly wise and unselfish guidance he provided to him during the very rewarding two years he spent as a Post-Doctoral Research Assistant at the University of East Anglia between 1989 and 1991.

\section{References}

[1] Smith, R.W., Applications of plate and scraped surface heat exchangers, Food Manufact., 47(10), 37-40 (1972).

[2] Trommelen, A.M. and Beek, W.J., Flow phenomena in a scraped-surface heat exchanger ("Votator"-type), Chem. Eng. Sci., 26, 1933-1942 (1971).

[3] Härröd, M., Methods to distinguish between laminar and vortical flow in scraped surface heat exchangers, J. Food Process Eng., 13, 39-57 (1990).

[4] Dumont, E., Fayolle, F. and Legrand, J., Flow regimes and wall shear rates determination within a scraped surface heat exchanger, J. Food Eng., 45, 195-207 (2000).

[5] Hall-Taylor, N.S., Scraped-surface heat exchangers - Introduction, Section 3.24 of Heat Exchanger Design Handbook 2008, Ed. G.F. Hewitt, Begell House Inc, Redding, CT, USA (2008).

[6] Russell, A.B., Burmester, S.S.H. and Winch, P.J., Characterization of shear thinning flow within a scraped surface heat exchanger, Trans IChemE - Part C: Food and Bioproducts Processing, 75(C3), 191-197 (1997).

[7] Wang, W., Walton, J.H. and McCarthy, K.L., Flow profiles of power law fluids in scraped surface heat exchanger geometry using MRI, J. Food Process Eng., 22, 11-27 (1999).

[8] Stranzinger, M., Feigl, K. and Windhab, E., Non-Newtonian flow behaviour in narrow annular gap reactors, Chem. Eng. Sci., 56, 3347-3363 (2001).

[9] Trommelen, A.M., Beek, W.J. and Van de Westelaken, H.C., A mechanism for heat transfer in a Votator-type scraped-surface heat exchanger, Chem. Eng. Sci., 26, 19872001 (1971).

[10] Qin, F.G.F., Chen, X.D. and Russell, A.B., Heat transfer at the subcooled-scraped surface with/without phase change, AIChE Journal, 49(8), 1947-1955 (2003).

[11] Trommelen, A.M. and Beek, W.J., The mechanism of power consumption in a Votator-type scraped-surface heat exchanger, Chem. Eng. Sci., 26, 1977-1986 (1971). 
[12] Sun, K.-H., Pyle, D.L., Fitt, A.D., Please, C.P., Baines, M.J. and Hall-Taylor, N., Numerical study of 2D heat transfer in a scraped surface heat exchanger, Comput. Fluid., 33, 869-880 (2004).

[13] Baccar, M. and Abid, M.S., Numerical analysis of three-dimensional flow and thermal behaviour in a scraped-surface heat exchanger, Rev. Gén. Therm., 36, 782-790 (1997).

[14] Baccar, M. and Abid, M.S., Simulation numérique des comportements hydrodynamiques et thermiques des échangeurs racleurs opérant en régime turbulent, Int. J. Therm. Sci., 38, 634-644 (1999).

[15] Sun, K.-H., Pyle, D.L., Baines, M.J., Hall-Taylor, N. and Fitt, A.D., Velocity profiles and frictional pressure drop for shear thinning materials in lid-driven cavities with fully developed axial flow, Chem. Eng. Sci., 61, 4697-4706 (2006).

[16] Fitt, A.D. and Please, C.P., Asymptotic analysis of the flow of shear-thinning foodstuffs in annular scraped heat exchangers, J. Eng. Math., 39, 345-366 (2001).

[17] Duffy, B.R., Wilson, S.K. and Lee, M.E.M., A mathematical model of fluid flow in a scraped-surface heat exchanger, J. Eng. Math., 57, 381-405 (2007).

[18] Fitt, A.D., Lee, M.E.M. and Please, C.P., Analysis of heat flow and "channelling" in a scraped-surface heat exchanger, J. Eng. Math., 57, 407-422 (2007).

[19] Rodriguez Pascual, M., Derksen, J.J., Van Rosmalen, G.M. and Witkamp, G.J., Flow and particle motion in scraped heat exchanger crystallizers, Chem. Eng. Sci., 64, 5153-5161 (2009).

[20] Härröd, M., Scraped surface heat exchangers: a literature survey of flow patterns, mixing effects, residence time distribution, heat transfer and power requirements, J. Food Process Eng., 9, 1-62 (1986).

[21] Rao, C.S. and Hartel, R.W., Scraped surface heat exchangers, Crit. Rev. Food Sci. Nutr., 46, 207-219 (2006).

[22] Wilson, S.K. and Duffy, B.R., On the gravity-driven draining of a rivulet of fluid with temperature-dependent viscosity down a uniformly heated or cooled substrate, J. Eng. Math., 42, 359-372 (2002). 


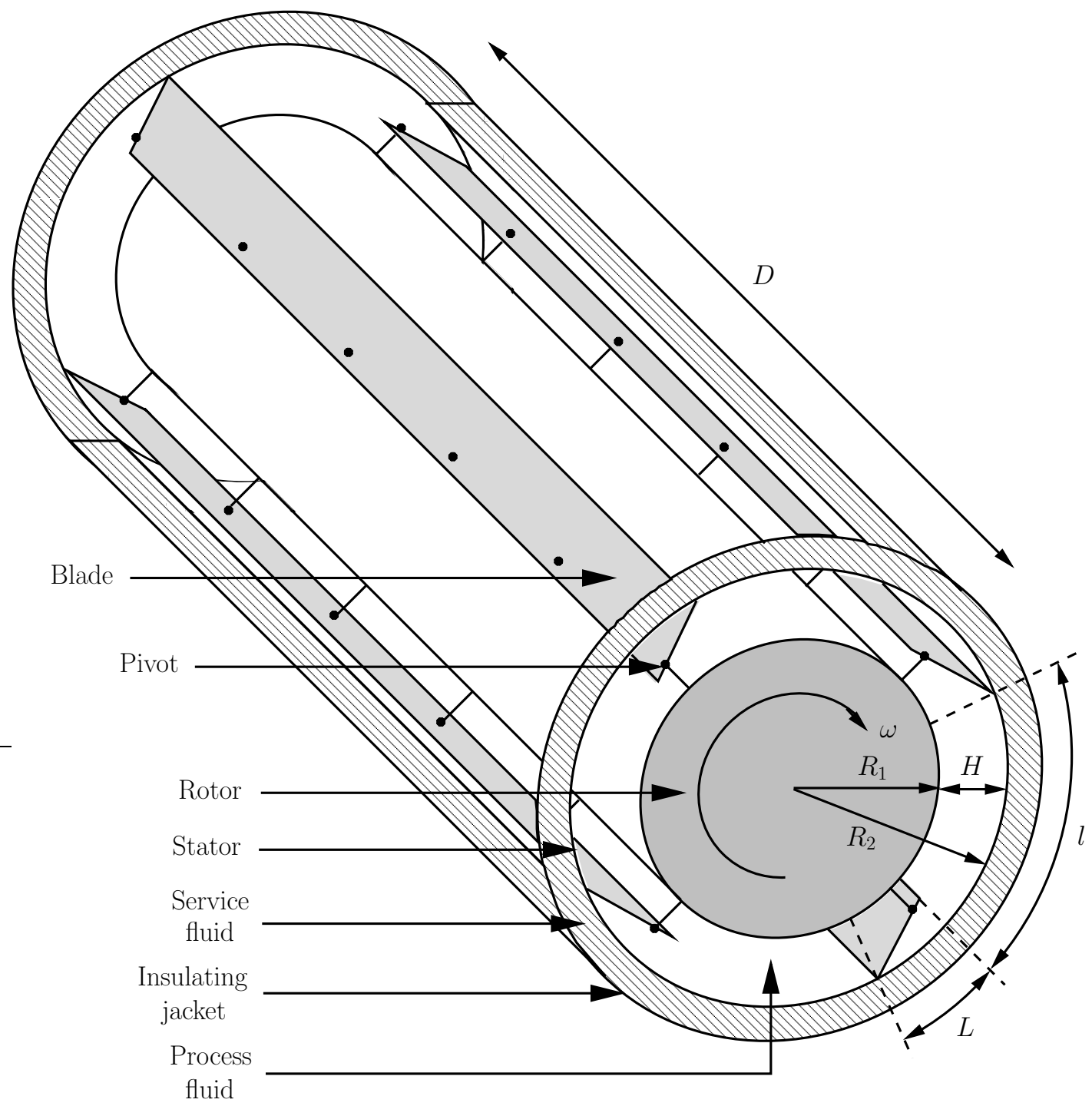

Figure 1: Cut-away schematic of a typical SSHE. 


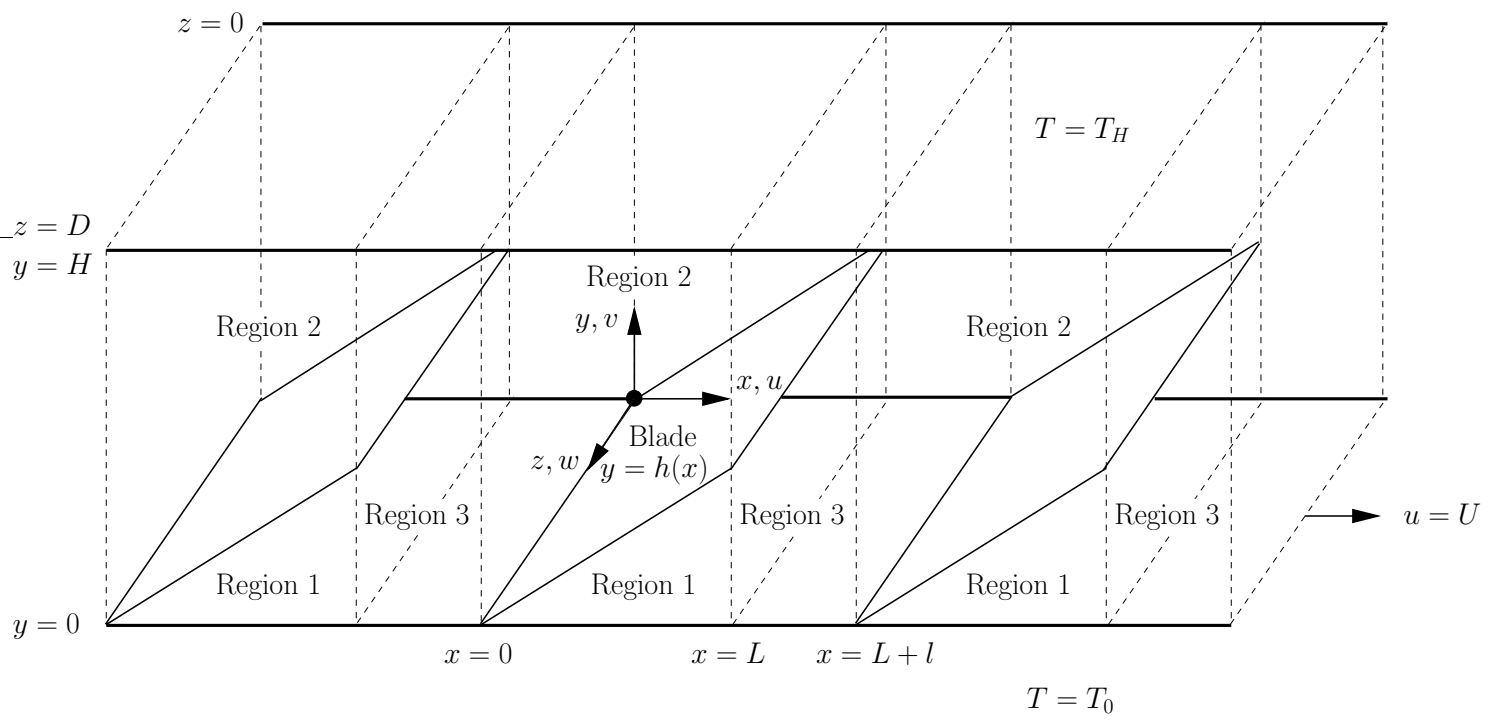

Figure 2: The geometry of the leading order mathematical model. 


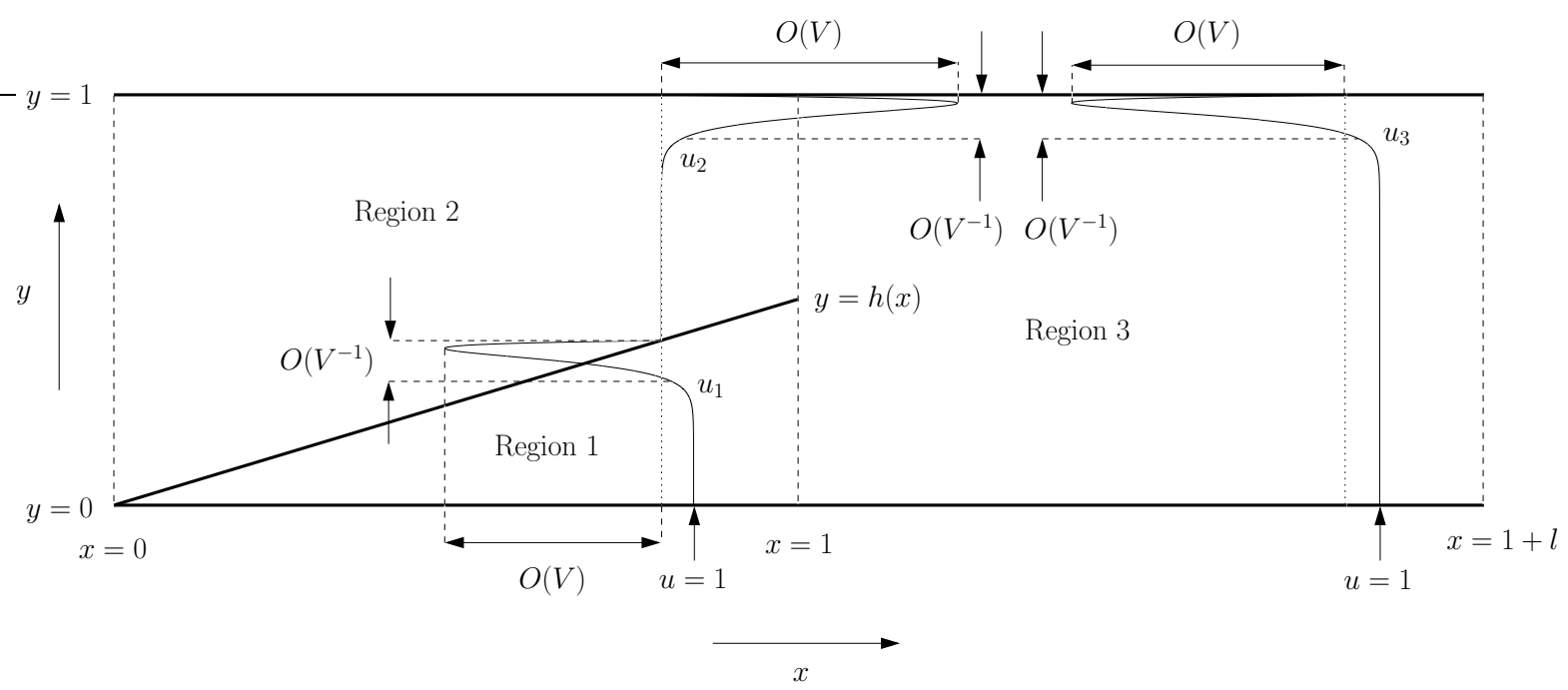

Figure 3: Sketches of the asymptotic solutions for the $x$ velocities in each of the three regions given by (86) and (90) in the limit $V \rightarrow \infty$. 


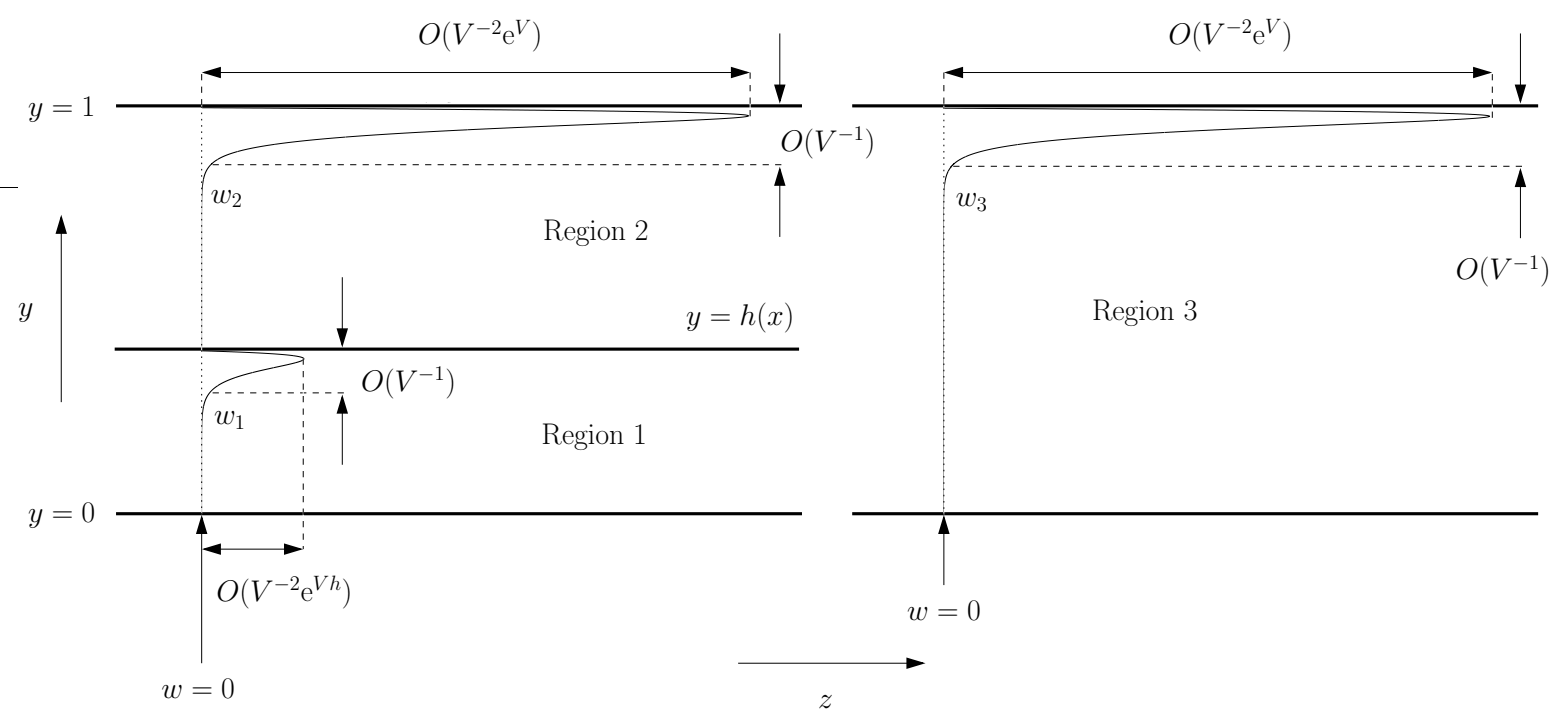

Figure 4: Sketches of the asymptotic solutions for the axial (i.e. the $z$ ) velocities in each of the three regions given by (91) in the limit $V \rightarrow \infty$. 


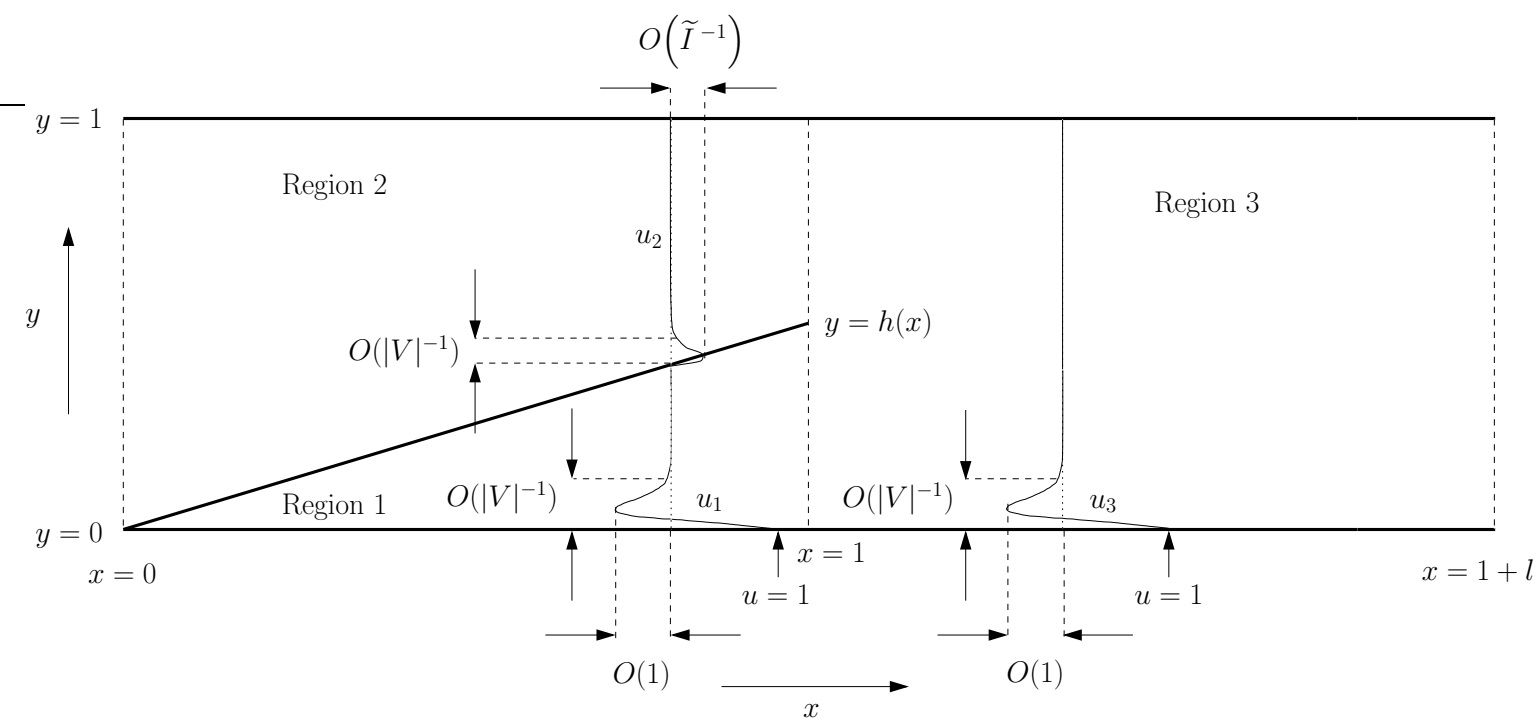

Figure 5: Sketches of the asymptotic solutions for the $x$ velocities in each of the three regions given by (95) and (100) in the limit $V \rightarrow-\infty$. 


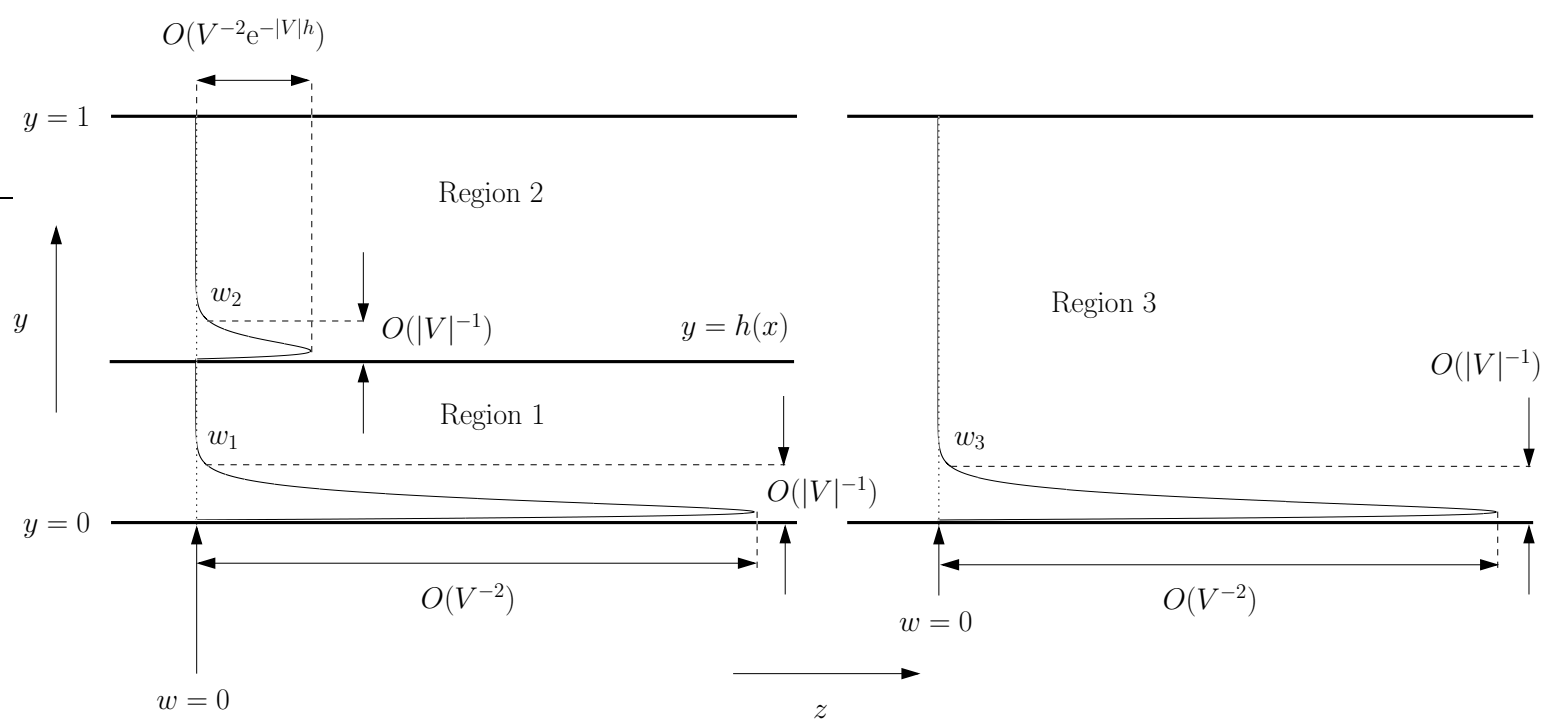

Figure 6: Sketches of the asymptotic solutions for the axial (i.e. the $z$ ) velocities in each of the three regions given by (101) in the limit $V \rightarrow-\infty$. 


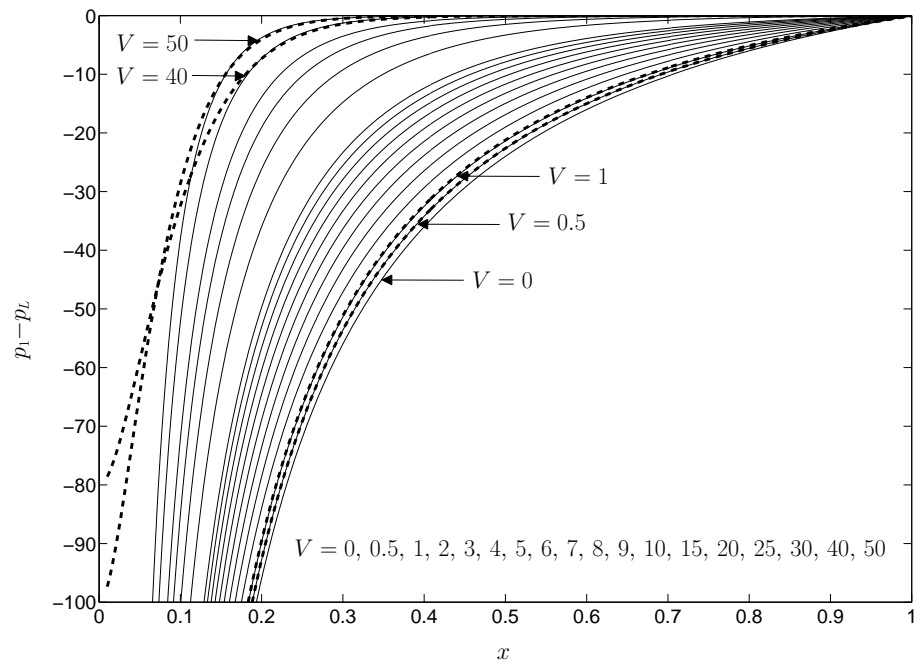

(a)

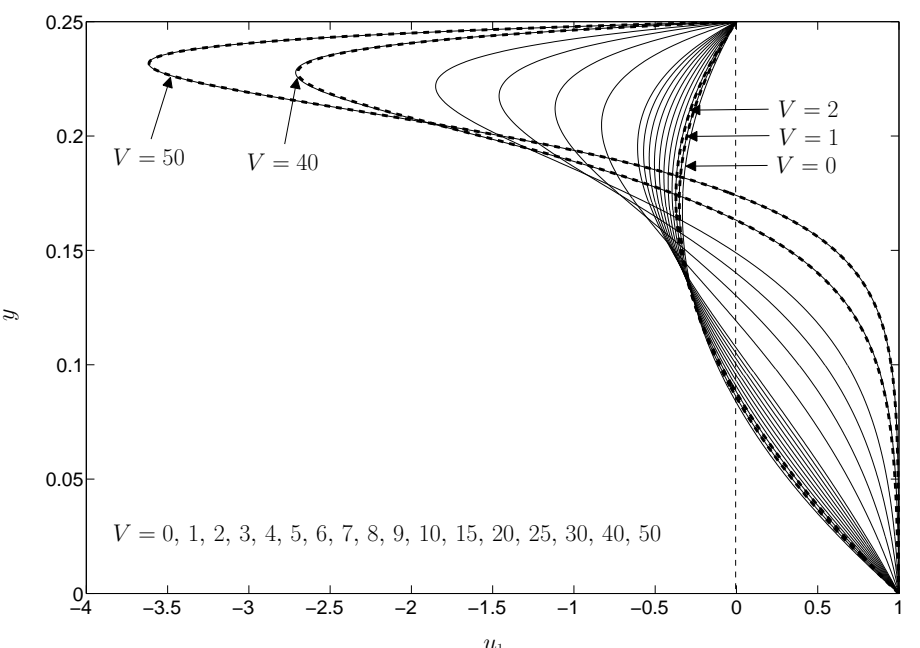

(b)

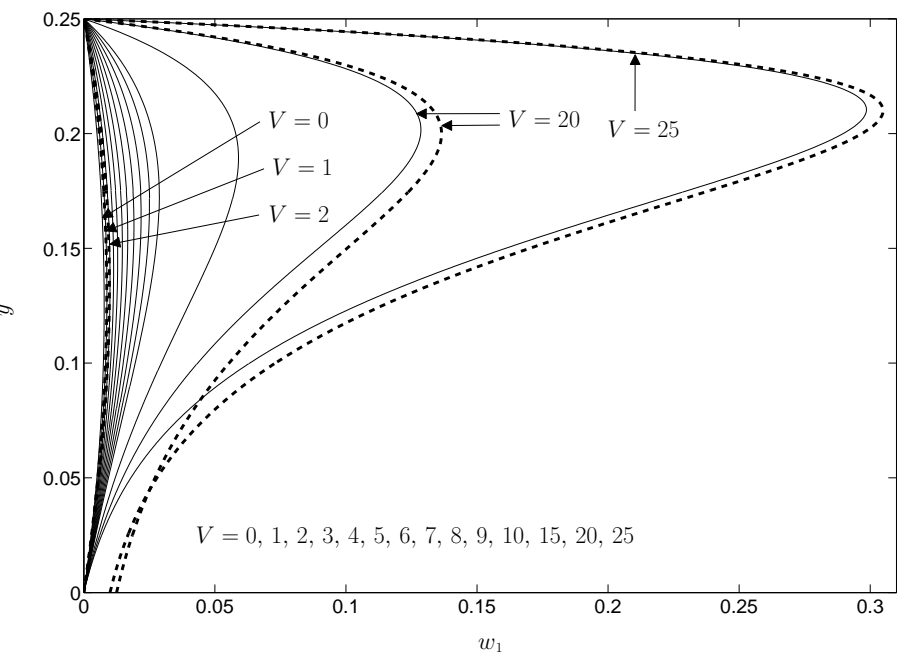

(c)

Figure 7: Plots of (a) the pressure difference $p_{1}-p_{L}$ as a function of $x$, (b) the $x$ velocity $u_{1}$ at $x=0.5$, and (c) the axial velocity $w_{1}$ at $x=0.5$, in region 1 for a range of positive values of the thermoviscosity number $V$ in the case $\alpha=0.5$ and $l=1$. The solid curves are the exact solutions obtained in Sec. 2.3, and the dashed curves are the small $V$ asymptotic solutions obtained in Sec. 4.1 and the large positive $V$ asymptotic solutions obtained in Sec. 4.2. 


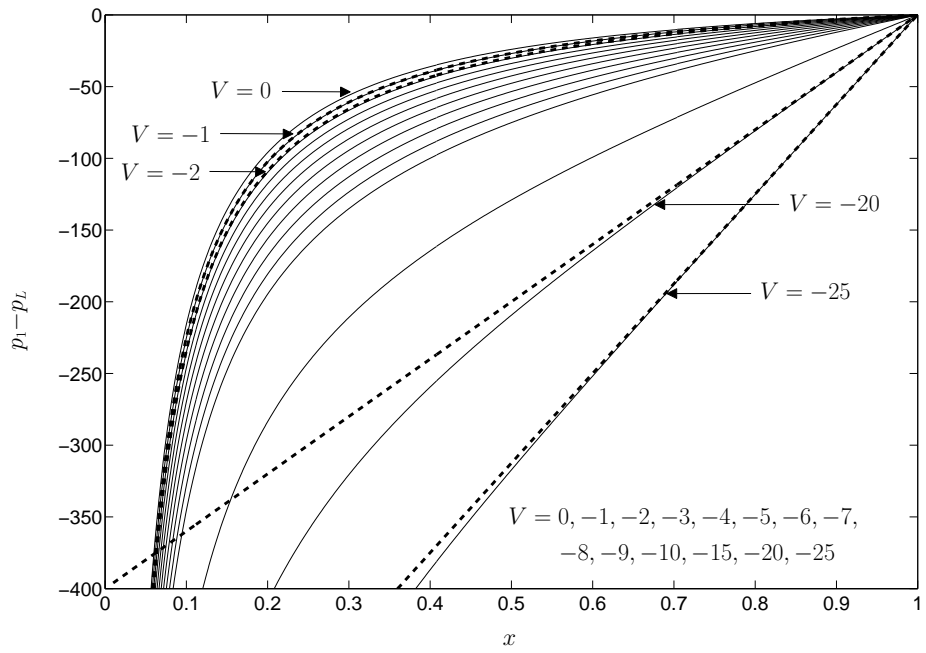

(a)

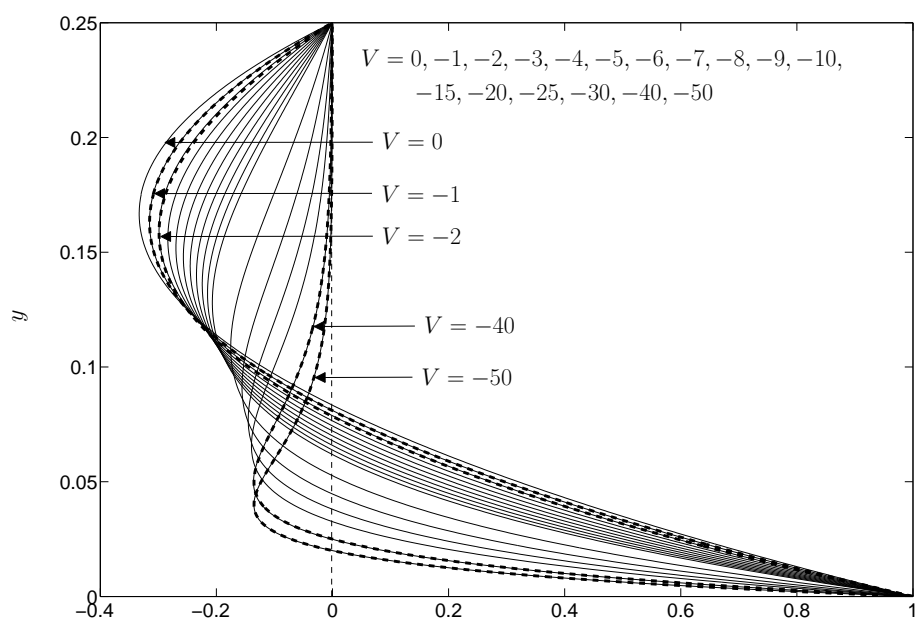

(b)
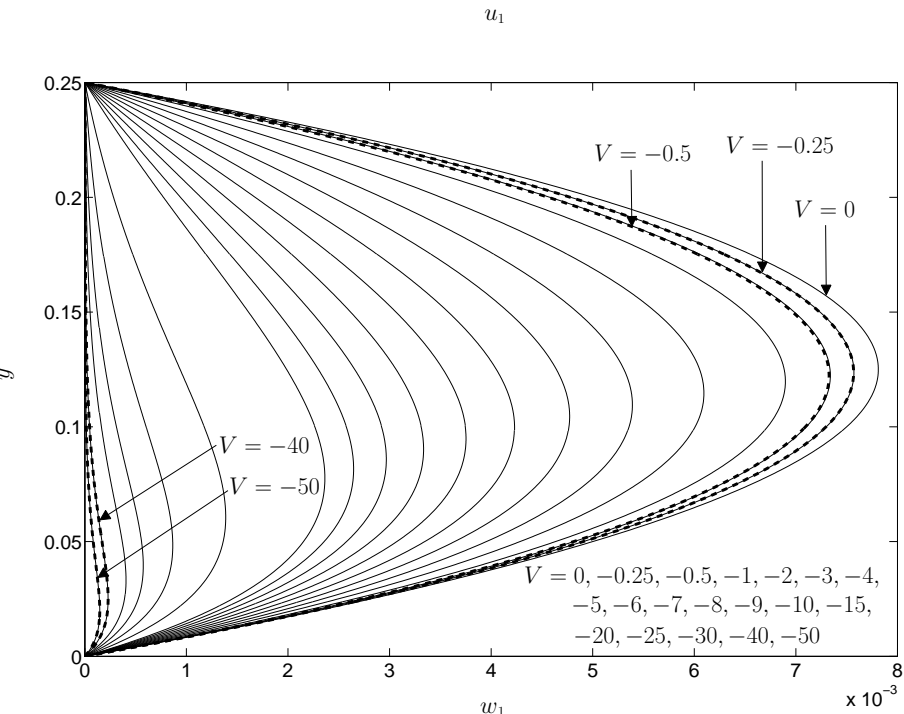

(c)

Figure 8: Plots of (a) the pressure difference $p_{1}-p_{L}$ as a function of $x$, (b) the $x$ velocity $u_{1}$ at $x=0.5$, and (c) the axial velocity $w_{1}$ at $x=0.5$, in region 1 for a range of negative values of the thermoviscosity number $V$ in the case $\alpha=0.5$ and $l=1$. The solid curves are the exact solutions obtained in Sec. 2.3, and the dashed curves are the small $V$ asymptotic solutions obtained in Sec. 4.1 and the large negative $V$ asymptotic solutions obtained in Sec. 4.3. 


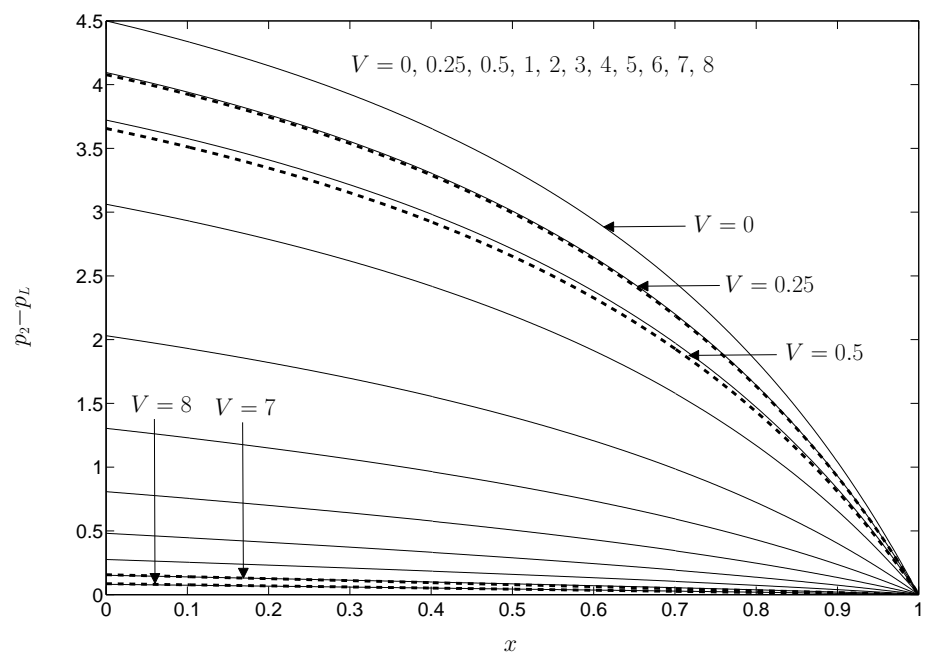

(a)

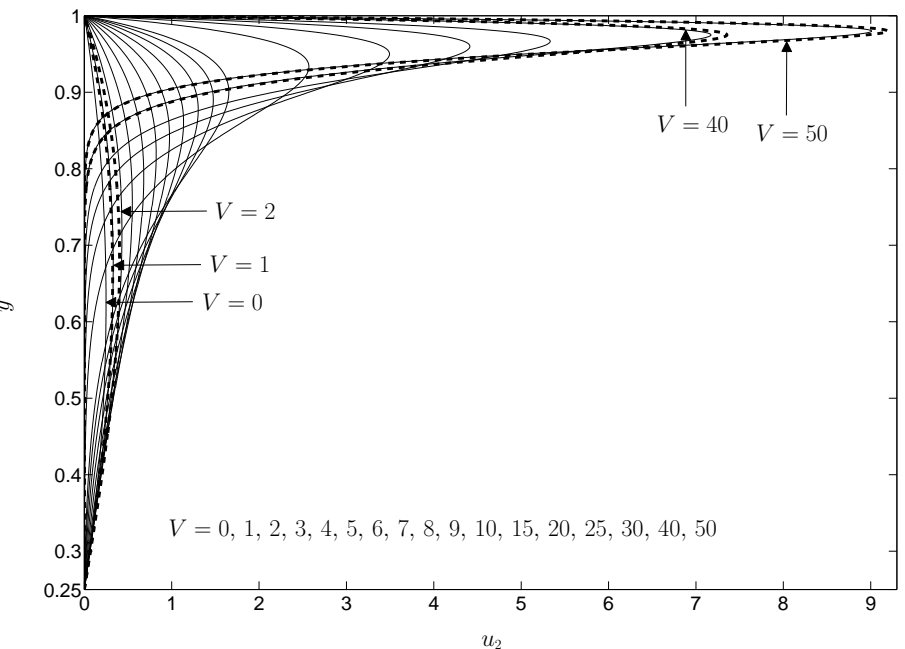

(b)

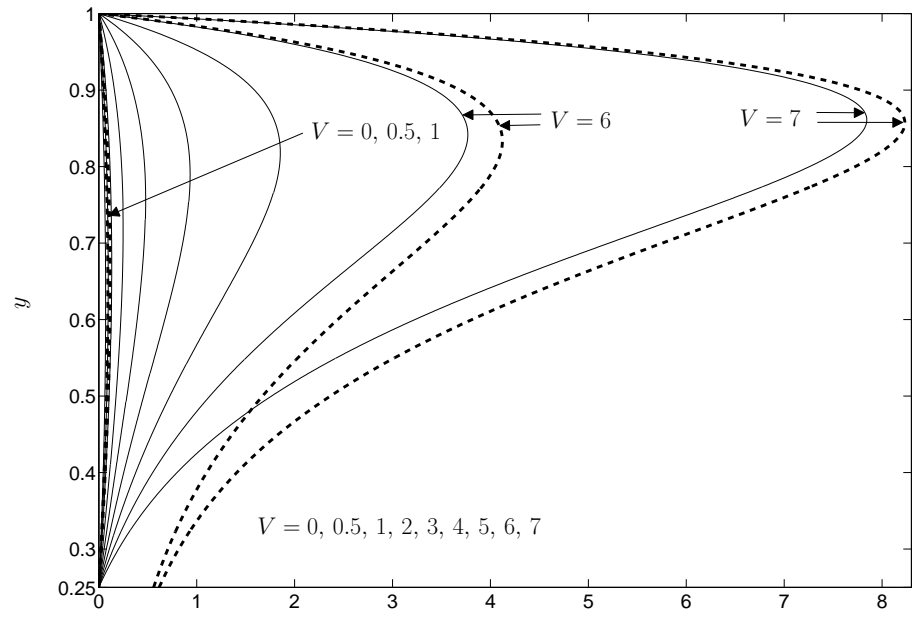

(c)

Figure 9: Plots of (a) the pressure difference $p_{2}-p_{L}$ as a function of $x$, (b) the $x$ velocity $u_{2}$ at $x=0.5$, and (c) the axial velocity $w_{2}$ at $x=0.5$, in region 2 for a range of positive values of the thermoviscosity number $V$ in the case $\alpha=0.5$ and $l=1$. The solid curves are the exact solutions obtained in Sec. 2.3, and the dashed curves are the small $V$ asymptotic solutions obtained in Sec. 4.1 and the large positive $V$ asymptotic solutions obtained in Sec. 4.2. 


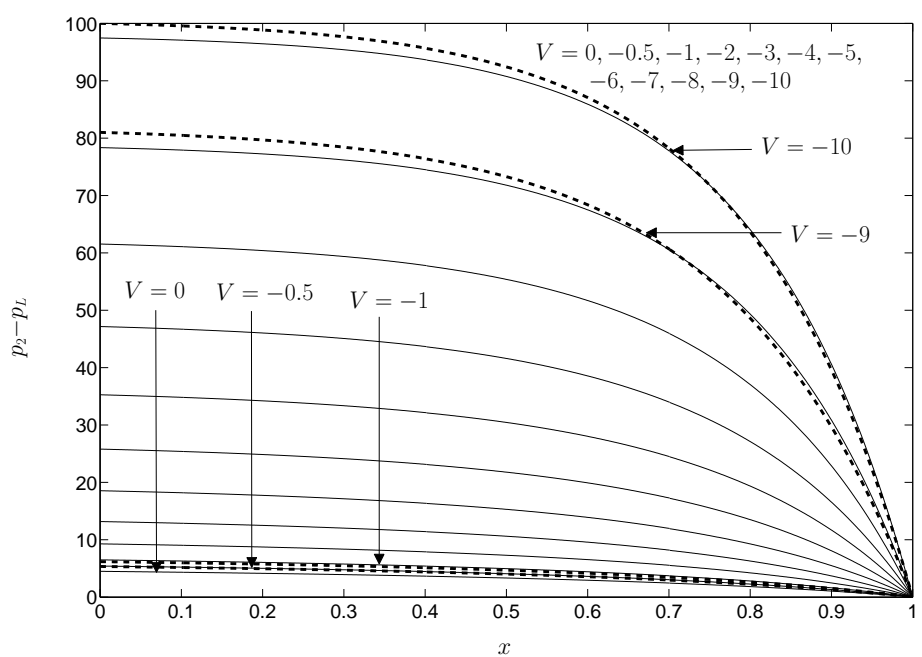

(a)

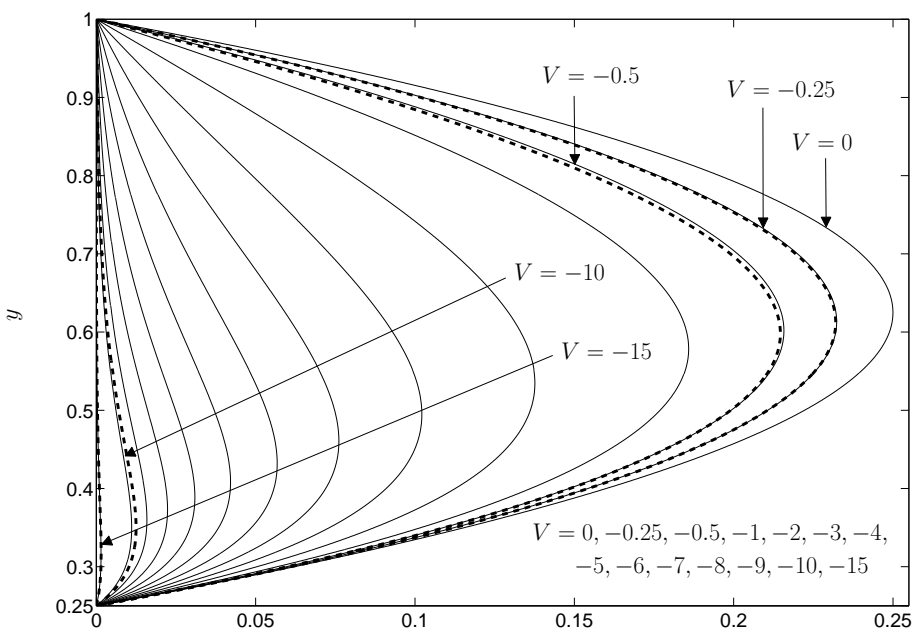

(b)

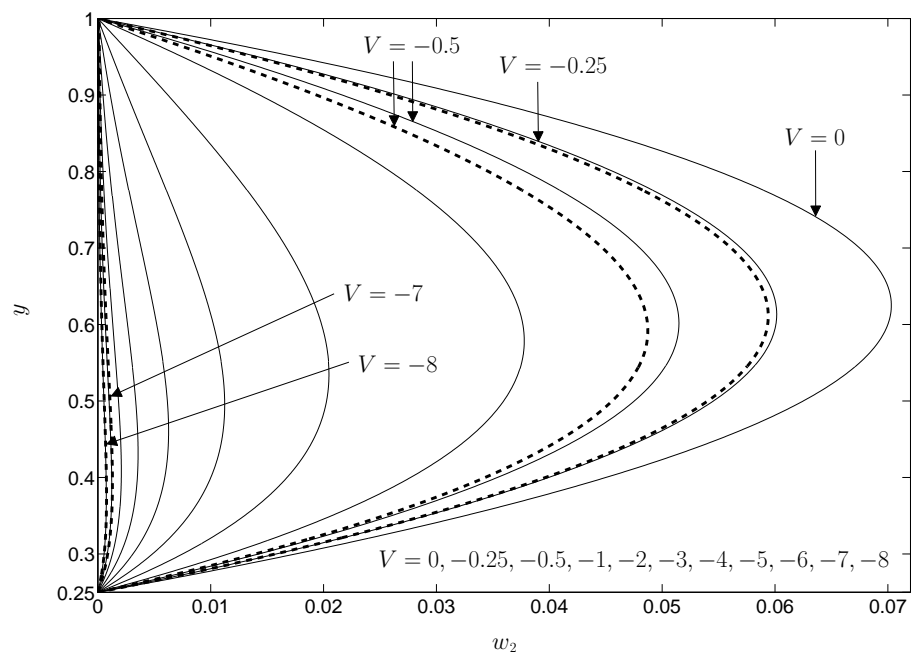

(c)

Figure 10: Plots of (a) the pressure difference $p_{2}-p_{L}$ as a function of $x$, (b) the $x$ velocity $u_{2}$ at $x=0.5$, and (c) the axial velocity $w_{2}$ at $x=0.5$, in region 2 for a range of negative values of the thermoviscosity number $V$ in the case $\alpha=0.5$ and $l=1$. The solid curves are the exact solutions obtained in Sec. 2.3, and the dashed curves are the small $V$ asymptotic solutions obtained in Sec. 4.1 and the large negative $V$ asymptotic solutions obtained in Sec. 4.3. 


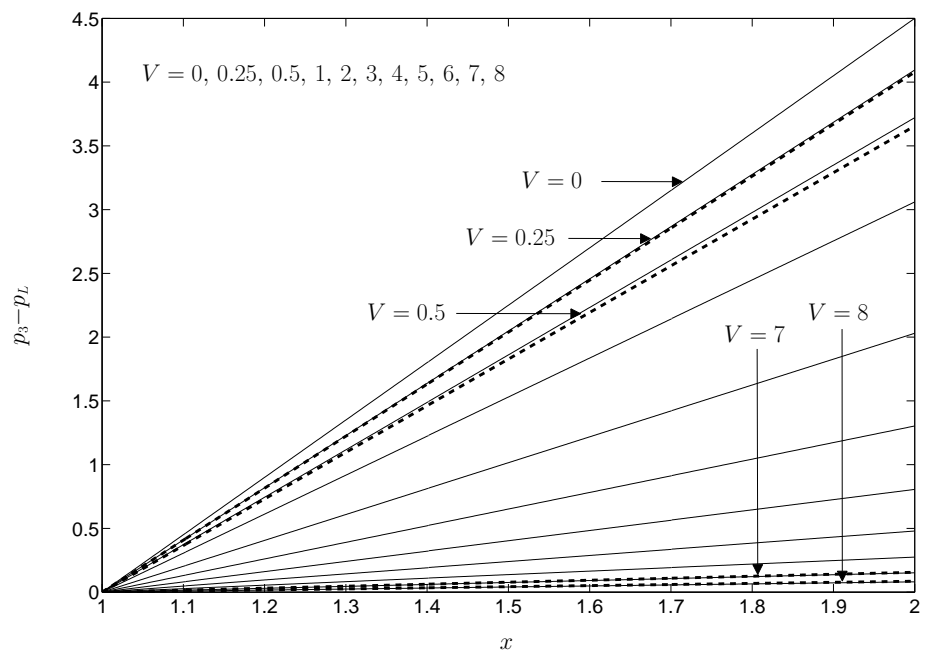

(a)

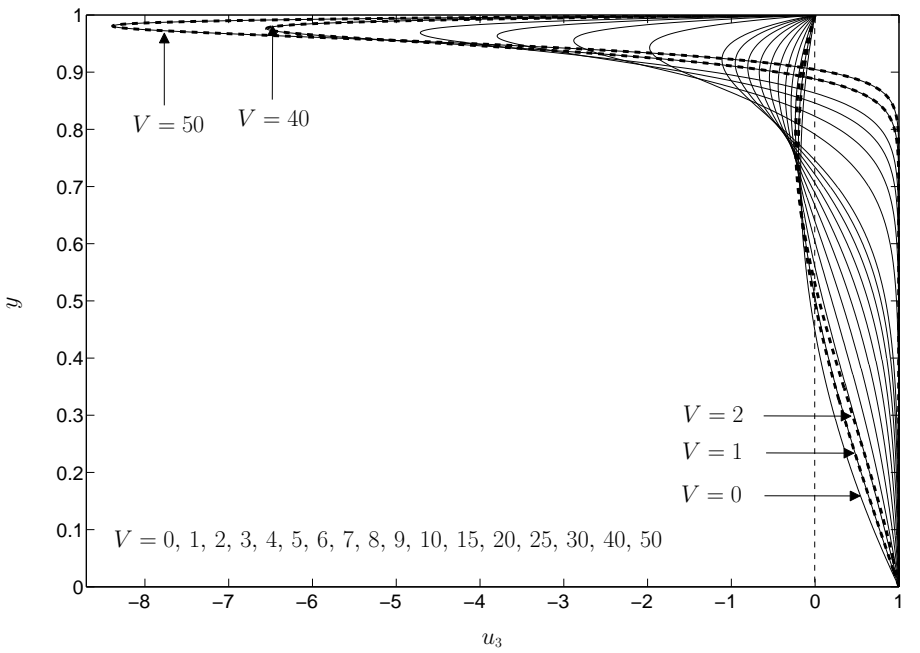

(b)

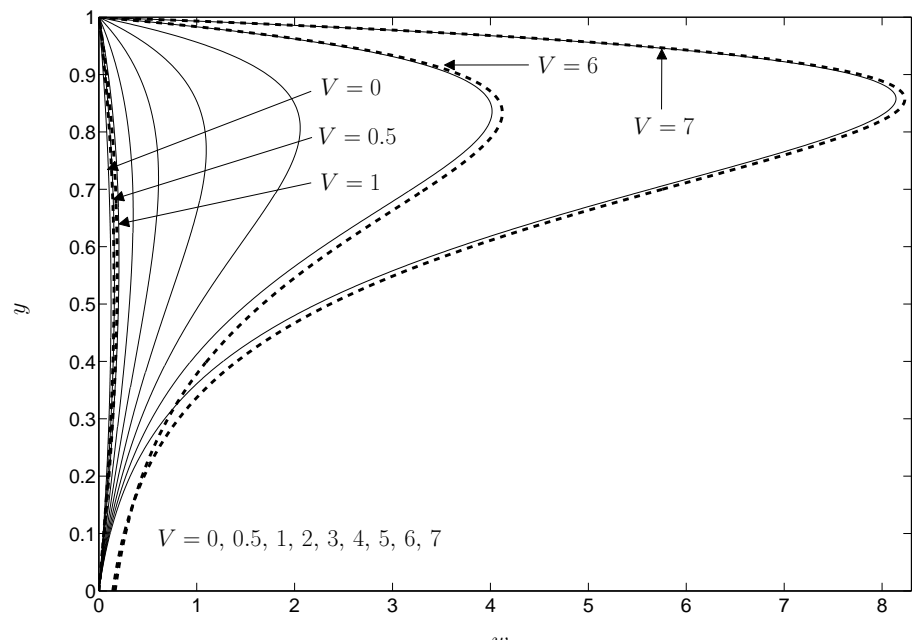

(c)

Figure 11: Plots of (a) the pressure difference $p_{3}-p_{L}$ as a function of $x$, (b) the $x$ velocity $u_{3}$ for $1 \leqslant x<1+l$, and (c) the axial velocity $w_{3}$ for $1 \leqslant x<1+l$, in region 3 for a range of positive values of the thermoviscosity number $V$ in the case $\alpha=0.5$ and $l=1$. The solid curves are the exact solutions obtained in Sec. 2.3, and the dashed curves are the small $V$ asymptotic solutions obtained in Sec. 4.1 and the large positive $V$ asymptotic solutions obtained in Sec. 4.2. 


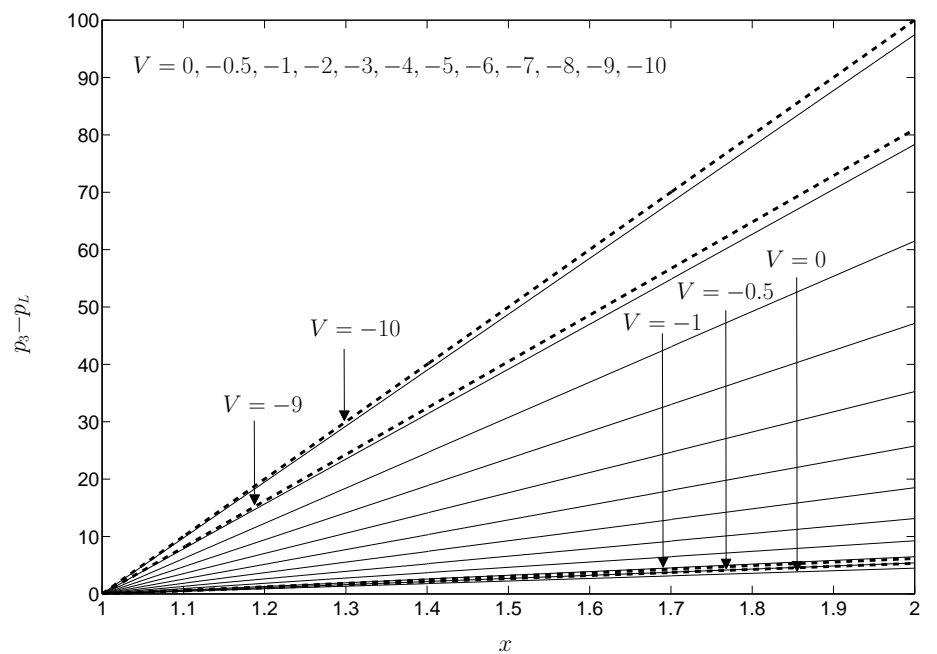

(a)

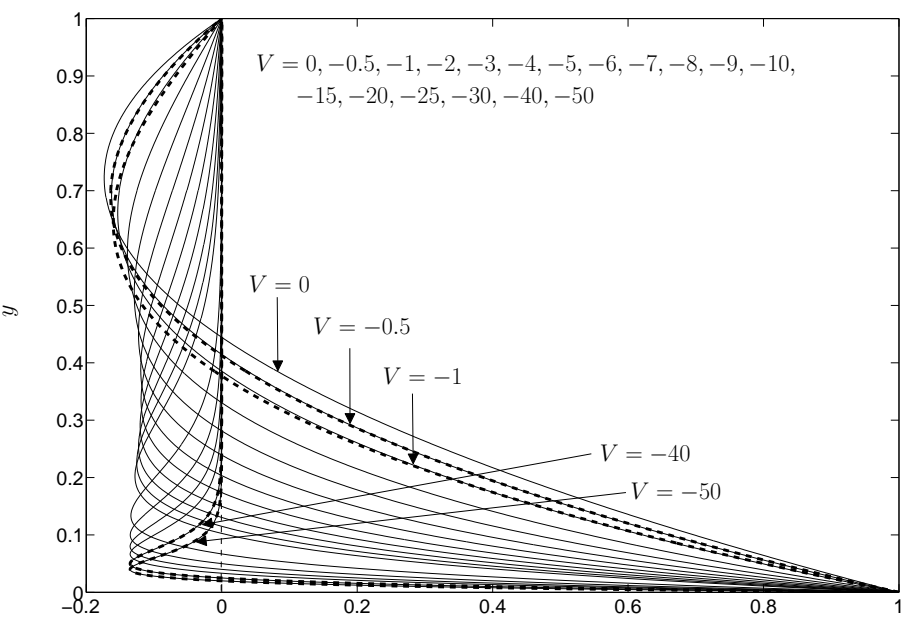

(b)

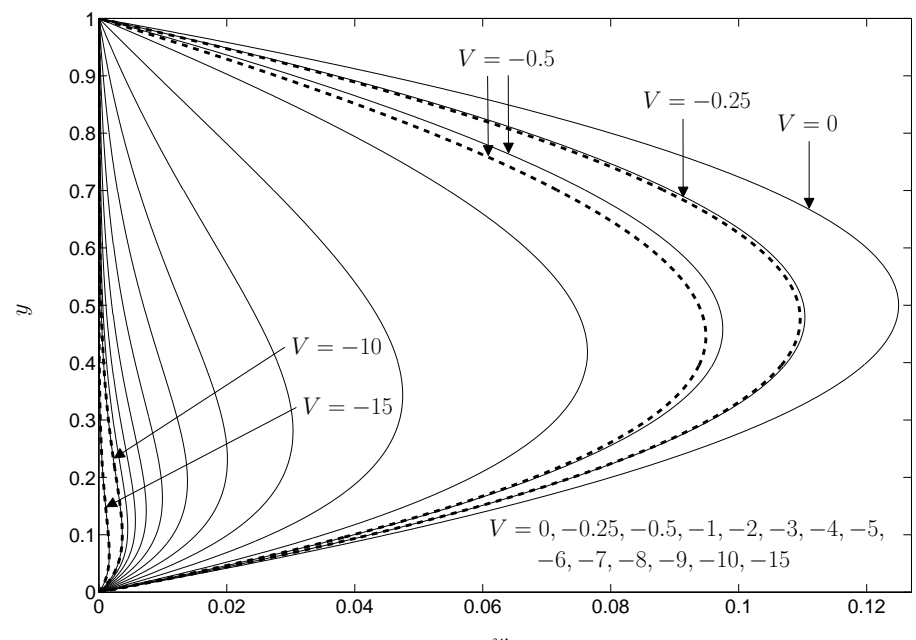

(c)

$w_{3}$

Figure 12: Plots of (a) the pressure difference $p_{3}-p_{L}$ as a function of $x$, (b) the $x$ velocity $u_{3}$ for $1 \leqslant x<1+l$, and (c) the axial velocity $w_{3}$ for $1 \leqslant x<1+l$, in region 3 for a range of negative values of the thermoviscosity number $V$ in the case $\alpha=0.5$ and $l=1$. The solid curves are the exact solutions obtained in Sec. 2.3, and the dashed curves are the small $V$ asymptotic solutions obtained in Sec. 4.1 and the large negative $V$ asymptotic solutions obtained in Sec. 4.3. 


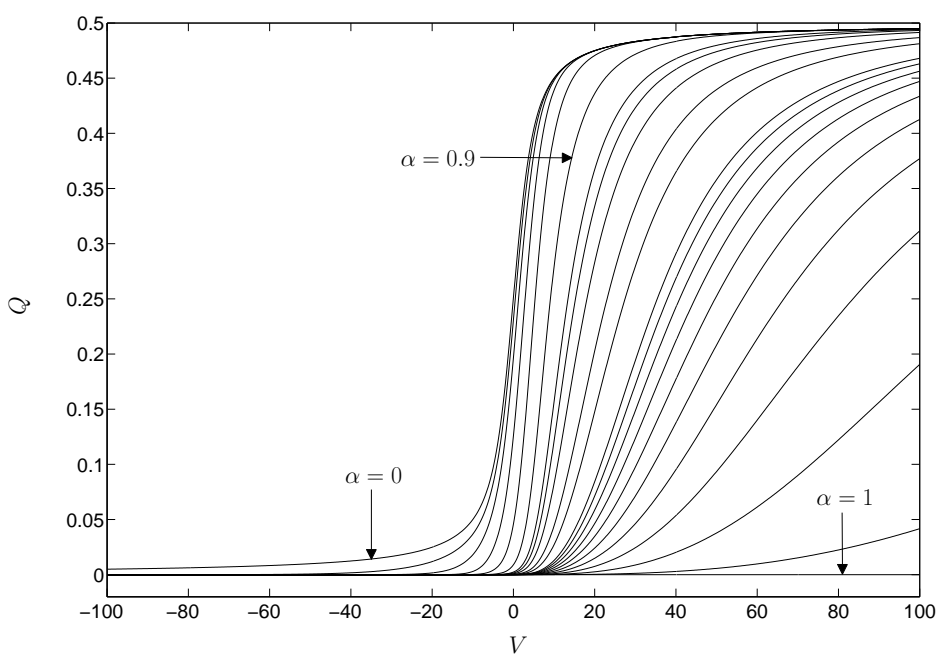

(a)

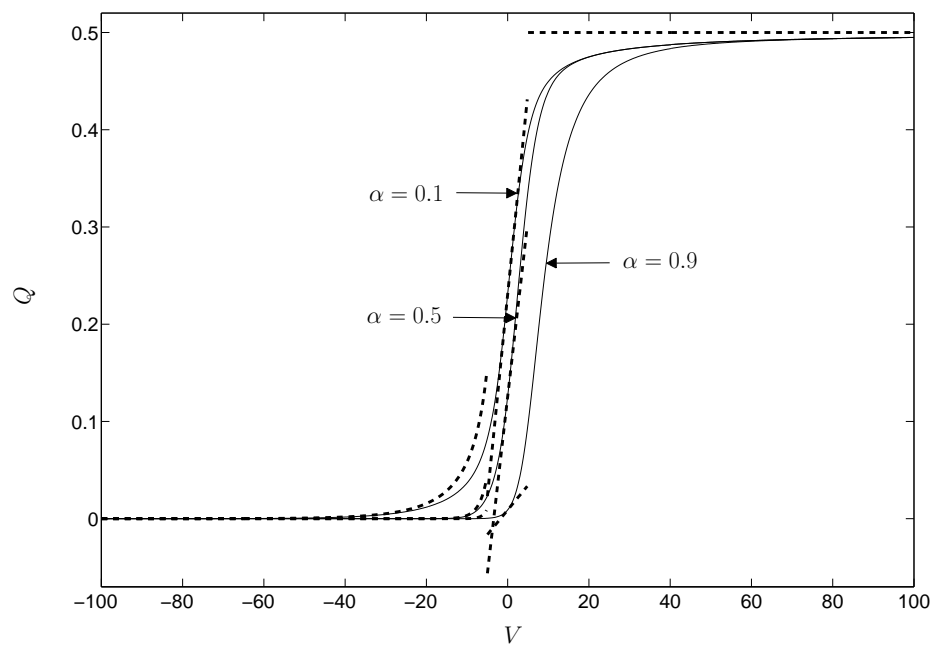

(b)

Figure 13: Plot of the transverse volume flux $Q$ given by (47) as a function of the thermoviscosity number $V$ for (a) $\alpha=0,0.1,0.25,0.5,0.75,0.9,0.95,0.96,0.97$, $0.98,0.985,0.99,0.991,0.992,0.993,0.994,0.995,0.996,0.997,0.998,0.999,1$ and (b) $\alpha=0.1,0.5,0.9$ in the case $l=1$. In (b) the solid curves are the exact solutions obtained in Sec. 2.3, and the dashed curves are the small $V$ asymptotic solutions obtained in Sec. 4.1 and the large positive and negative $V$ asymptotic solutions obtained in Secs 4.2 and 4.3 . 


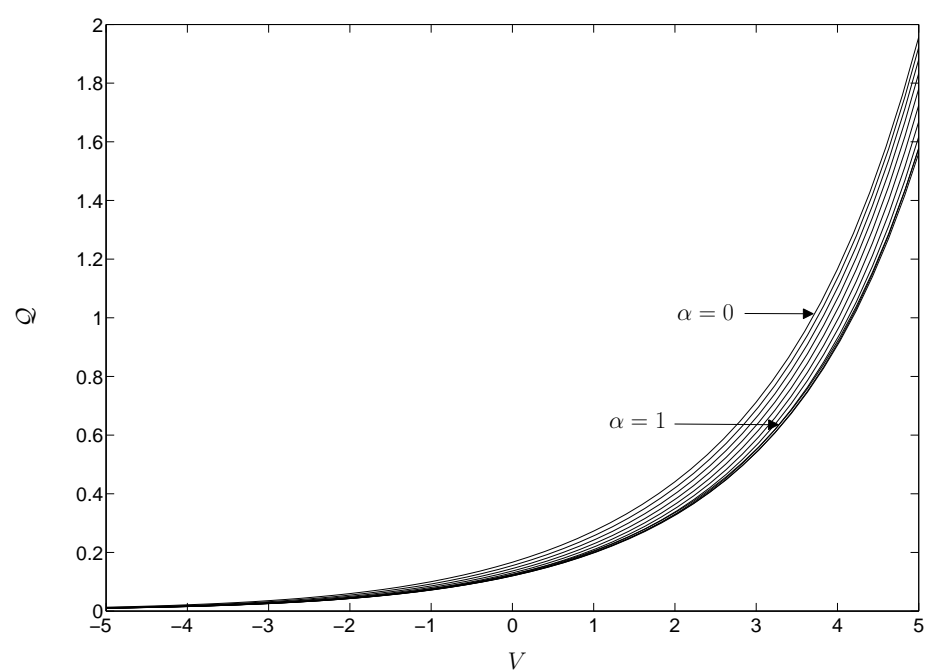

(a)

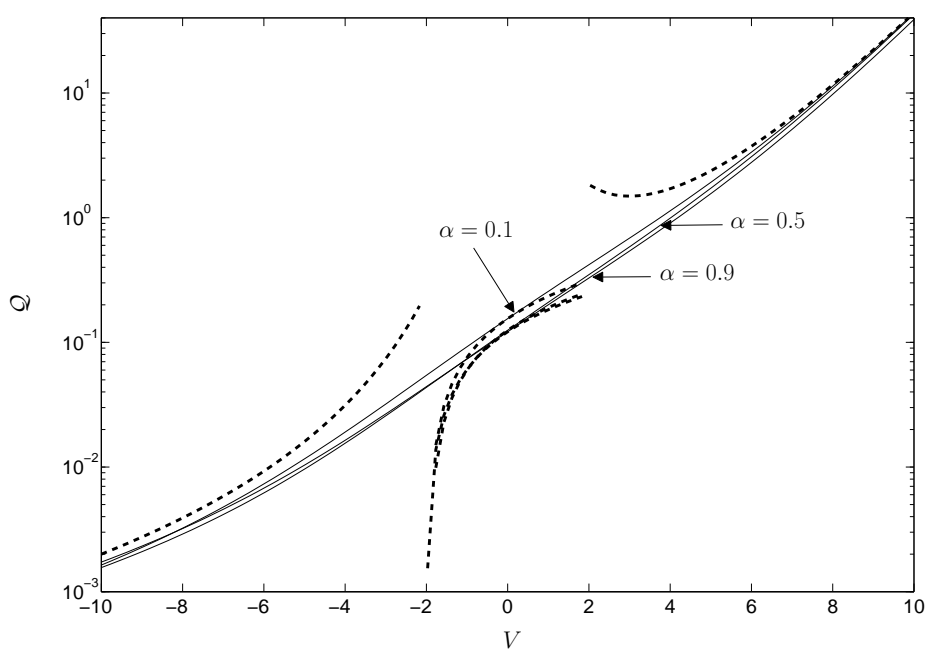

(b)

Figure 14: Plot of the axial volume flux $\mathcal{Q}$ given by (51) as a function of the thermoviscosity number $V$ for (a) $\alpha=0,0.1,0.2,0.3,0.4,0.5,0.6,0.7,0.8,0.9,1$ and (b) $\alpha=0.1$, $0.5,0.9$ in the case $l=1$. In (b) the solid curves are the exact solutions obtained in Sec. 2.3, and the dashed curves are the small $V$ asymptotic solutions obtained in Sec. 4.1 and the large positive and negative $V$ asymptotic solutions obtained in Secs 4.2 and 4.3 . 


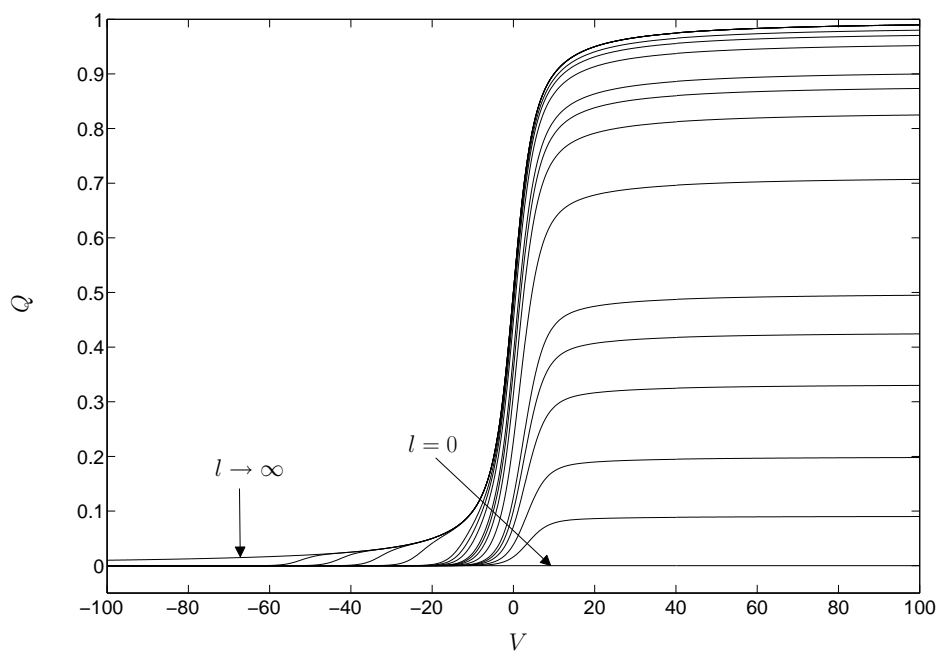

(a)

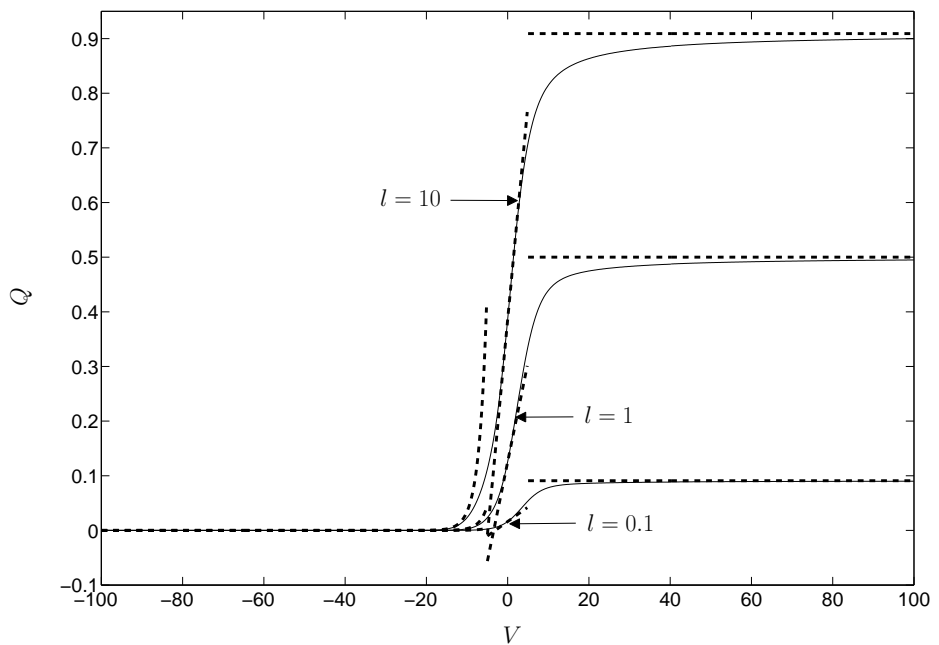

(b)

Figure 15: Plot of the transverse volume flux $Q$ given by (47) as a function of the thermoviscosity number $V$ for blade separation lengths (a) $l=0,0.1,0.25,0.5,0.75,1$, $2.5,5,7.5,10,25,50,100,1 \times 10^{4}, 1 \times 10^{6}, 1 \times 10^{8}, 1 \times 10^{10}, \infty$ and (b) $l=0.1,1,10$ in the case $\alpha=0.5$. In (b) the solid curves are the exact solutions obtained in Sec. 2.3, and the dashed curves are the small $V$ asymptotic solutions obtained in Sec. 4.1 and the large positive and negative $V$ asymptotic solutions obtained in Secs 4.2 and 4.3. 


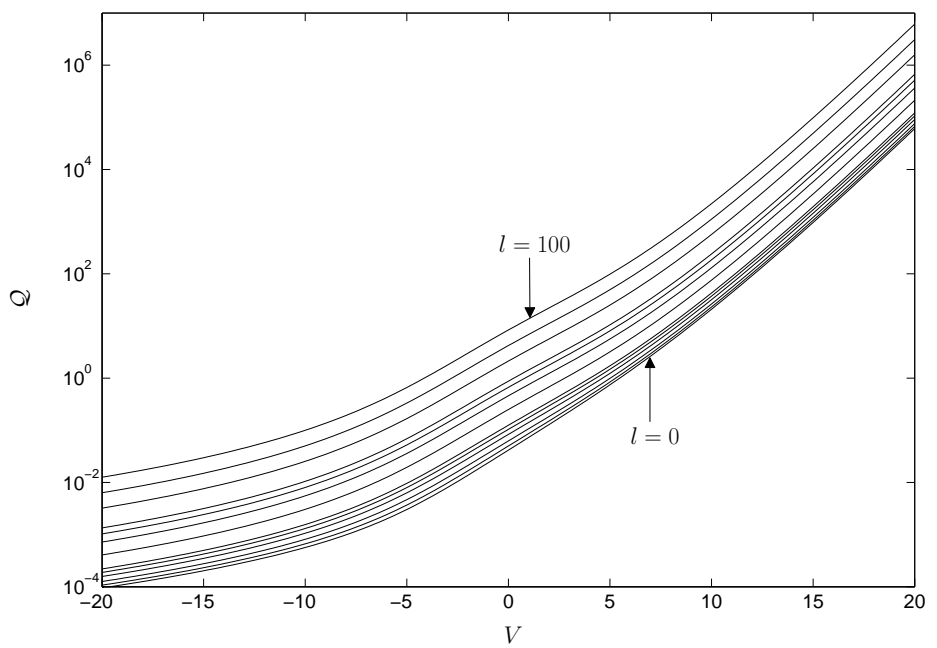

(a)

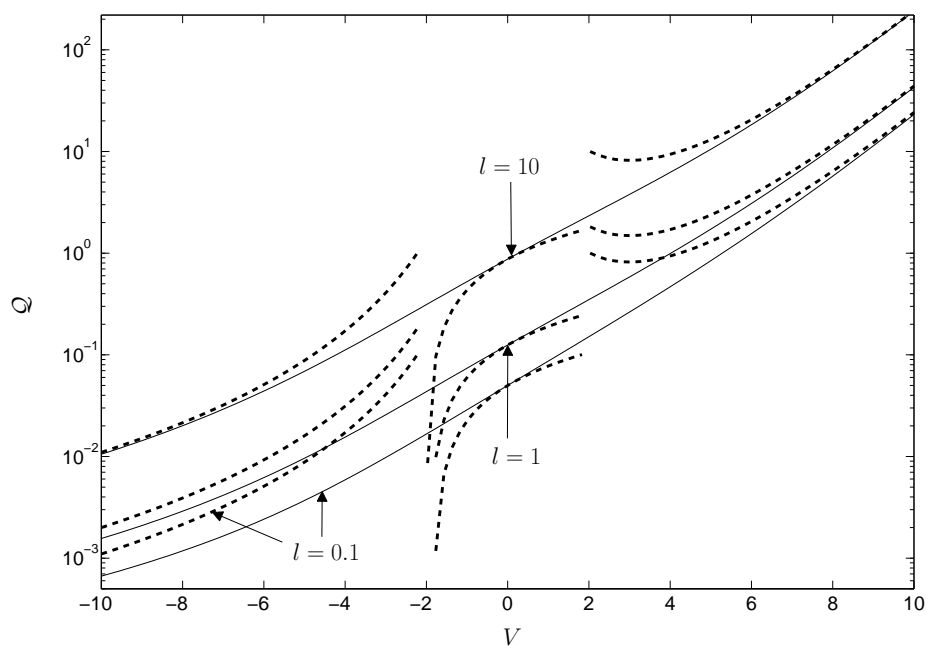

(b)

Figure 16: Plot of the axial volume flux $\mathcal{Q}$ given by (51) as a function of the thermoviscosity number $V$ for blade separation lengths (a) $l=0,0.1,0.25,0.5,0.75,1,2.5,5,7.5$, $10,25,50,100$ and (b) $l=0.1,1,10$ in the case $\alpha=0.5$. In (b) the solid curves are the exact solutions obtained in Sec. 2.3, and the dashed curves are the small $V$ asymptotic solutions obtained in Sec. 4.1 and the large positive and negative $V$ asymptotic solutions obtained in Secs 4.2 and 4.3 . 